\title{
RENORMALISATION OF PAIR CORRELATION MEASURES FOR PRIMITIVE INFLATION RULES AND ABSENCE OF ABSOLUTELY CONTINUOUS DIFFRACTION
}

\author{
MICHAEL BAAKE, FRANZ GÄHLER, AND NEIL MAÑIBO
}

\begin{abstract}
The pair correlations of primitive inflation rules are analysed via their exact renormalisation relations. We introduce the inflation displacement algebra that is generated by the Fourier matrix of the inflation and deduce various consequences of its structure. Moreover, we derive a sufficient criterion for the absence of absolutely continuous diffraction components, as well as a necessary criterion for its presence. This is achieved via estimates for the Lyapunov exponents of the Fourier matrix cocycle of the inflation rule. We also discuss some consequences for the spectral measures of such systems. While we develop the theory first for the classic setting in one dimension, we also present its extension to primitive inflation rules in higher dimensions with finitely many prototiles up to translations.
\end{abstract}

\section{INTRODUCTION}

The spectral structure of substitution systems gives valuable insight into such systems and their mutual relations. However, with our present knowledge, it is still rather far to a classification in sufficient generality. While the general Pisot substitution conjecture, despite great progress in recent years (see [1,39] and references therein), is still open, the class of constant-length substitutions is essentially understood, at least on an algorithmic level. In fact, building on [51], Bartlett [15] presented a general method how to determine the spectral measure of maximal type computationally, for any given primitive constant-length substitution. Remarkably, this approach also works in higher dimensions, and various general results have been derived from it.

From the viewpoint of diffraction theory, it is also possible to derive the spectral type, because the required spectral measures can be realised as the restriction of certain diffraction measures to a fundamental domain [12]. More generally, as long as one works with systems with pure point spectrum, it does not matter whether one considers the dynamical or the diffraction spectrum, as pure pointedness of one implies the other, without restriction to constant-length substitutions; see $[42,10,12]$.

In general, the situation is less favourable when considering substitutions that are not of constant length. For instance, beyond the much-studied Pisot substitution case, one additional topological obstacle emerges when the substitution matrix has eigenvalues of modulus $|\lambda| \geqslant 1$ other than the leading one. Here, it generally matters [22] whether one considers the symbolic dynamical system, under the $\mathbb{Z}$-action of the shift, or the geometric one, the latter defined via tiles (intervals) of natural length and studied under the natural translation action 
of $\mathbb{R}$. As was realised and demonstrated in [3], the geometric version possesses an exact renormalisation identity for the pair correlation measures of the system. This gives access to some spectral properties that, to our knowledge, are presently not available on the symbolic level. Substitutions of constant length are special in the sense that the two viewpoints, symbolic and geometric, coincide, which ultimately is the reason for their better accessibility.

In this paper, we develop the renormalisation approach for the geometric setting of primitive substitutions in more generality, building on previous work on several classes of examples $[3,46,8]$. Moreover, we extend the approach to inflation tilings of finite local complexity (FLC) in Euclidean spaces of arbitrary dimension. In fact, we also take first steps to go beyond the FLC case. We employ the diffraction theory approach and derive consequences for the spectral measures where presently possible. Our particular interest is the derivation of sufficient criteria for the absence of absolutely continuous diffraction and spectral measures, as well as necessary criteria for their presence. This is motivated by the rare occurrence of such components, which to date is essentially limited to Rudin-Shapiro-type sequences and their generalisations; compare [29, 5, 20, 21] and references therein.

It will be instrumental for our analysis that we formulate various aspects on the symbolic level, while the core of our analysis rests on the natural geometric realisation in order to profit from the inherent self-similarity in the form of exact renormalisation relations; see $[18,19]$ for related results on spectral measures of one-dimensional systems via the analysis of matrix Riesz products. Here, to make the distinction between the types of dynamical systems as transparent as possible, we will speak of substitution rules on the symbolic side, but of inflation rules on its geometric counterpart, thus following the notation and terminology of $[5,6]$. For convenience, various results are briefly recalled from there. Rather than repeating the proofs, we provide precise references instead.

The paper is organised as follows. After recalling some general facts about unbounded, but translation-bounded measures on $\mathbb{R}$ and their Fourier transforms in Section 2, we extend the notions and results of [3] on the classic Fibonacci case to general primitive inflation rules in Section 3. Here, we introduce the inflation displacement algebra and derive some of its general properties in relation to the Fourier matrix of the inflation and its cocycle, which will later help to understand the Lyapunov spectrum of this cocycle, in Section 4. Some additional material on the corresponding Kronecker product algebra [3, 2] is gathered in an appendix. We then introduce the pair correlation functions and their renormalisation relations, which are a consequence of the inflation structure. They lead to an infinite-dimensional system (9) of linear equations, with nevertheless an essentially unique solution (Theorem 3.19).

A measure-theoretic reformulation leads to the pair correlation measures and their Fourier transforms, with specific access to their spectral components. In Theorem 3.23, the structure of the pure point part is made explicit in analogy to the Bombieri-Taylor approach, compare [43], while the ensuing analysis of the absolutely continuous parts forms the core of our paper. Here, via the introduction of certain Lyapunov exponents for the Fourier matrix cocycle, we derive an effective sufficient criterion for the absence of absolutely continuous components in 
the diffraction measure of the inflation system (Theorem 3.28), together with a general upper bound for the maximal exponent in Theorem 3.34. This also provides a necessary criterion for the presence of absolutely continuous diffraction components in Corollary 3.35.

In Section 4, we apply the general theory to the class of Abelian bijective substitutions of constant length. With some input from group representation and character theory, we can derive the known absence $[51,15]$ of absolutely continuous spectral components, both in the diffraction and in the dynamical sense, in an independent way in Theorems 4.1 and 4.7. This is also illustrated with several examples, which are selected to highlight the relations between substitutions, Fourier matrices and Lyapunov exponents.

Finally, in Section 5, we extend our approach to inflation tilings in higher dimensions with finitely many prototiles up to translations. As we shall briefly indicate, this already admits the treatment of some tilings without finite local complexity [7]. The key observation for primitive inflation tilings with finitely many translational prototiles is that the approach with the Fourier matrices readily generalises and leads to the essential separation of the geometric structure of the tiling in Euclidean space from the combinatorial data of the inflation rule (and its geometry via the pair correlation measures). This ultimately leads to a criterion for the absence of absolutely continuous spectral components in Theorem 5.7, which is the extension of Theorem 3.28 to this situation. We demonstrate the effectiveness of our criterion by treating two examples, namely binary block substitutions (in Section 5.4) and the planar Godrèche-Lançon-Billard tiling (in Section 5.5). The latter is a planar non-Pisot inflation tiling, built with the Penrose rhombuses, and is shown, via the aforementioned method, to have an essentially singular continuous diffraction spectrum.

\section{RADON MEASURES AND EBERLEIN CONVOLUTIONS}

Here, we recall some notions and results on unbounded measures that we shall need throughout. A (complex) Radon measure on $\mathbb{R}^{d}$ is a continuous linear functional on the space $C_{\mathrm{c}}(\mathbb{R})$ of continuous functions with compact support, the latter equipped with the inductive limit topology. By the general Riesz-Markov theorem, Radon measures correspond to regular Borel measures on $\mathbb{R}^{d}$, and we shall use this connection several times. Note that these need not be finite measures. If $\mu$ is a measure, its twisted counterpart $\widetilde{\mu}$ is defined via $\widetilde{\mu}(g)=\overline{\mu(\widetilde{g})}$ for $g \in C_{\mathrm{c}}(\mathbb{R})$, where $\widetilde{g}(x):=\overline{g(-x)}$. Moreover, given a mapping $f$ of $\mathbb{R}^{d}$ into itself, the

push-forward of $\mu$, denoted by $f . \mu$, is defined by $(f . \mu)(g):=\mu(g \circ f)$ where $g \in C_{\mathrm{c}}\left(\mathbb{R}^{d}\right)$, often called test function from now on.

The convolution of two finite measures $\mu$ and $\nu$ is defined as

$$
(\mu * \nu)(g)=\int_{\mathbb{R}^{d}} \int_{\mathbb{R}^{d}} g(x+y) \mathrm{d} \mu(x) \mathrm{d} \nu(y) .
$$

When $f$ is a linear map on $\mathbb{R}^{d}$, one has the relation

$$
f \cdot(\mu * \nu)=(f \cdot \mu) *(f . \nu),
$$


as follows from a simple calculation; compare [7, Lemma 2.3]. Let us fix an averaging sequence $\mathcal{R}=\left(R_{n}\right)_{n \in \mathbb{N}}$ of compact sets $R_{n} \subset \mathbb{R}^{d}$ with $R_{n} \subset R_{n+1}^{\circ}$ and $\bigcup_{n \in \mathbb{N}} R_{n}=\mathbb{R}^{d}$. We will assume throughout that $\mathcal{R}$ is a van Hove sequence; see $[5,13]$ for details. Now, with $\left.\mu\right|_{R_{n}}$ denoting the restriction of $\mu$ to $R_{n}$, the Eberlein (or volume-averaged) convolution of two translationbounded measures, relative to $\mathcal{R}$, is defined as

$$
\mu \circledast \nu:=\lim _{n \rightarrow \infty} \frac{\left.\left.\mu\right|_{R_{n}} * \nu\right|_{R_{n}}}{\operatorname{vol}\left(R_{n}\right)},
$$

provided the limit exists (we shall not consider any other situation below).

A Radon measure $\mu$ is called positive definite, if $\mu(g * \widetilde{g}) \geqslant 0$ holds for all $g \in C_{\mathrm{c}}\left(\mathbb{R}^{d}\right)$. A positive and positive definite measure is automatically translation bounded [16, Prop. 4.4]. An important instance of this is the autocorrelation measure $\gamma$ of a translation-bounded measure $\omega$, defined as

$$
\gamma=\gamma_{\omega}:=\omega \circledast \widetilde{\omega} .
$$

Provided the Eberlein convolution exists, which will be true in all cases studied below, $\gamma$ is a positive definite measure, and in many later situations, it is also a positive measure; see [5, Sec. 8.5] and references therein for more. For the study of spectral properties, we are then interested in the Fourier transform of $\gamma$, denoted as $\widehat{\gamma}$, which is known as the diffraction measure of $\omega$; see $[37,11]$ as well as $[5$, Ch. 9] for general background.

There are several possibilities to define and analyse the Fourier transform of a measure. This is a non-trivial issue, see [49] for a systematic exposition, and part of our later analysis will rely on the existence of the Fourier transform. We use a standard version of the Fourier transform $[53,16]$ that, for integrable functions on $\mathbb{R}^{d}$ viewed as Radon-Nikodym densities, reads

$$
\widehat{f}(k)=\int_{\mathbb{R}^{d}} \mathrm{e}^{-2 \pi \mathrm{i} k x} f(x) \mathrm{d} x,
$$

where $k x$ denotes the standard inner product between $k$ and $x$ in $\mathbb{R}^{d}$. Any positive definite measure is Fourier transformable. The Fourier transform of a positive definite measure is a positive measure; see [16, Ch. I.4] or [5, Sec. 8.6] for details. Moreover, Fourier transform is continuous on the class of positive and positive definite measures.

Lemma 2.1. Let $\mu, \nu$ be translation-bounded measures such that $\mu \circledast \widetilde{\nu}$ as well as $\mu \circledast \widetilde{\mu}$ and $\nu \circledast \widetilde{\nu}$ exist, all with respect to the same averaging sequence $\mathcal{R}$. Then, $\mu \circledast \widetilde{\nu}$ is a translationbounded and transformable measure, as is $\widetilde{\mu} \circledast \nu$.

Proof. Observe first that $\widetilde{\mu} \circledast \nu=\widetilde{\mu \circledast \widetilde{\nu}}$, wherefore it suffices to prove the claim for $\mu \circledast \widetilde{\nu}$. Now, as a variant of the (complex) polarisation identity, one verifies that

$$
\mu \circledast \widetilde{\nu}=\frac{1}{4} \sum_{\ell=1}^{4} \mathrm{i}^{\ell}\left(\mu+\mathrm{i}^{\ell} \nu\right) \circledast\left(\mu+\mathrm{i}^{\ell} \nu\right)^{\sim},
$$


where all measures on the right-hand side exist due to our assumptions. Consequently, $\mu \circledast \widetilde{\nu}$ is a complex linear combination of four positive definite measures, each of which is transformable. Moreover, due to our assumptions, these four measures are translation bounded, so $\mu \circledast \widetilde{\nu}$ is translation bounded and transformable as well.

If $\omega$ is a positive definite measure, $\widehat{\omega}$ is a well-defined positive measure that has a unique Lebesgue decomposition $\widehat{\omega}=\widehat{\omega}_{\mathrm{pp}}+\widehat{\omega}_{\text {cont }}$ into a pure point measure, with a supporting set that is at most countable, and a continuous one. On the level of $\omega$ itself, this corresponds to the Eberlein decomposition $\omega=\omega_{\text {sap }}+\omega_{0 \text {-wap }}$ into a strongly almost periodic measure, whose Fourier transform is $\widehat{\omega}_{\mathrm{pp}}$, and a null-weakly almost periodic one; see [49,6] for background. Unfortunately, for the further decomposition $\widehat{\omega}_{\text {cont }}=\widehat{\omega}_{\mathrm{sc}}+\widehat{\omega}_{\mathrm{ac}}$ into the singular continuous and absolutely continuous parts, no general counterpart in the Eberlein decomposition is known at present. However, some special cases have recently been analysed by Strungaru [58] that look promising.

\section{Primitive inflation Rules in one Dimension}

Let $\mathcal{A}=\left\{a_{1}, \ldots, a_{n_{a}}\right\}$ be our alphabet with $n_{a}$ symbols or letters, and let $\varrho: a_{i} \mapsto \varrho\left(a_{i}\right)$ be a primitive substitution rule with substitution matrix $M_{\varrho}=\left(M_{i j}\right)_{1 \leqslant i, j \leqslant n_{a}}$, where

$$
M_{i j}:=\text { number of letters of type } a_{i} \text { in } \varrho\left(a_{j}\right)
$$

as usual; compare $[51,5]$. We will also use the notation $\varrho=\left(\varrho\left(a_{1}\right), \ldots, \varrho\left(a_{n_{a}}\right)\right)$ for $\varrho$. Let $\lambda_{\mathrm{PF}}$ denote the Perron-Frobenius (PF) eigenvalue of $M$, with the usual interpretation that the corresponding right eigenvector, in statistical normalisation, provides the relative frequencies of the letters in a fixed point of $\varrho$ (or of a suitable power of it), and that the left eigenvector contains the natural prototile lengths (up to a common overall factor) for the corresponding geometric inflation rule; see [5, Ch. 4] and references therein for background. In short, each inflation step consists in first expanding each tile by a factor of $\lambda$ and then dissecting it into tiles of the original size, in the order specified by $\varrho$. By slight abuse of notation, we use the symbol $\varrho$ both for the (symbolic) substitution rule and for its partner, the (geometric) inflation rule.

Starting from a fixed point tiling $\mathcal{T}$ of $\mathbb{R}$ under the inflation rule (or one of its powers, if necessary), the corresponding compact hull is defined as $\mathbb{Y}=\overline{\{t+\mathcal{T} \mid t \in \mathbb{R}\}}$, with the closure being taken in the local topology. By standard results, see [5] and references therein, one obtains a topological dynamical system $(\mathbb{Y}, \mathbb{R})$ that is strictly ergodic. ${ }^{1}$ In other words, there is just one way to put an invariant probability measure $\mu$ on it, which is the one induced by the patch frequencies, and the resulting measure-theoretic dynamical system is denoted by $(\mathbb{Y}, \mathbb{R}, \mu)$. Given any element from $\mathbb{Y}$, which is a tiling of $\mathbb{R}$ by $n_{a}$ (possibly coloured) intervals, there is a corresponding Delone set $\Lambda$ obtained by taking the left endpoints of all

\footnotetext{
${ }^{1}$ Note that there is another topological dynamical system, denoted by $(\mathbb{X}, \mathbb{Z})$, which emerges from the shift action on the symbolic hull $\mathbb{X}$, the latter obtained as the orbit closure of a symbolic fixed point of $\varrho$ or a suitable power of it; see [5] for more. Also this system is strictly ergodic.
} 
tiles of $\mathcal{T}$. If the tile lengths are not distinct, we distinguish them by colour, and do the same for the points. Then, one has a unique decomposition $\Lambda=\bigcup_{i=1}^{n_{a}} \Lambda_{i}$, so that the (coloured) tiling $\mathcal{T}$ and the (coloured) point set $\Lambda$ are mutually locally derivable from each other, or MLD for short; see [5] for the concept and more background. It is clear that $\mathcal{T}$ and $\Lambda$ define topologically conjugate dynamical systems on the orbit closure, which we tacitly identify from now on for ease of presentation.

3.1. The inflation displacement algebra (IDA). Given $\varrho$, we now assume that we have chosen prototiles of natural length, hence proportional to the entries of the left PF eigenvector of $M_{\varrho}$. For standardisation, we shall often take the shortest interval to have unit length. Now, define the displacement matrix $T=\left(T_{i j}\right)_{1 \leqslant i, j \leqslant n_{a}}$ with set-valued entries

$$
T_{i j}:=\left\{\text { all relative positions of intervals of type } a_{i} \text { in the patch } \varrho\left(a_{j}\right)\right\} .
$$

For simple examples of non-constant length, we refer to [2, Sec. 4.2] and [8, Sec. 3.1]. Here and below, relative positions are always defined via the left endpoints of the tiles (intervals) or patches. Note that $\varrho\left(a_{j}\right)$ is a level-1 supertile. With these definitions, we have $\operatorname{card}(T):=$ $\left(\operatorname{card}\left(T_{i j}\right)\right)_{1 \leqslant i, j \leqslant n_{a}}=M_{\varrho}$. Let us also define the total set $S_{T}:=\bigcup_{i, j} T_{i j}$ of all relative positions of prototiles in level-1 supertiles. Since they are all non-negative by construction, we may write $S_{T}$ as an ordered set,

$$
S_{T}=\left\{x_{1}, \ldots, x_{m}\right\}
$$

with $0=x_{1}<x_{2}<\ldots<x_{m}$ and some $m \in \mathbb{N}$.

Definition 3.1. Let $T_{i j}$ be the displacement sets from Eq. (3). Then, for $k \in \mathbb{R}$, the Fourier matrix $B(k)=\left(B_{i j}(k)\right)_{1 \leqslant i, j \leqslant n_{a}}$ of $\varrho$ is defined by

$$
B_{i j}(k)=\sum_{t \in T_{i j}} \mathrm{e}^{2 \pi \mathrm{i} t k}
$$

Clearly, one has $\overline{B(k)}=B(-k)$ for all $k \in \mathbb{R}$. Note that $B(0)=M_{\varrho}$, and that we have a decomposition of the form

$$
B(k)=\sum_{x \in S_{T}} \mathrm{e}^{2 \pi \mathrm{i} k x} D_{x}
$$

with integer matrices $D_{x}$ that satisfy $\sum_{x \in S_{T}} D_{x}=M_{\varrho}$, as is clear from setting $k=0$. Note that $D_{x}=D_{y}$ for $x \neq y$ is possible. Since at most one prototile of a patch can have its left endpoint at a given position, it is clear that any $D_{x}$ can only have entries 0 and 1 , namely

$$
D_{x, i j}= \begin{cases}1, & \text { if } \varrho\left(a_{j}\right) \text { contains a tile of type } a_{i} \text { at position } x \\ 0, & \text { otherwise. }\end{cases}
$$

These matrices are a generalisation of what is known as digit matrices in constant-length substitutions [61, 30], wherefore we adopt the name here as well; compare also [51, Ch. VIII], where they appear as instruction matrices.

Let us next consider the $\mathbb{C}$-algebra $\mathcal{B}$ that is generated by the one-parameter matrix family $\{B(k) \mid k \in \mathbb{R}\}$. Since the algebra $\mathcal{B}$ is also a finite-dimensional vector space over $\mathbb{C}$, it 
is automatically closed (in any of the matrix norms, which are all equivalent in this finitedimensional setting).

Fact 3.2. The $\mathbb{C}$-algebra $\mathcal{B}$ that is generated by the matrix family $\{B(k) \mid k \in \mathbb{R}\}$ equals the $\mathbb{C}$-algebra $\mathcal{B}_{D}$ that is generated by the digit matrices $\left\{D_{x} \mid x \in S_{T}\right\}$.

Proof. The inclusion $\mathcal{B} \subseteq \mathcal{B}_{D}$ is immediate from Eq. (4). For the converse, let us first assume that the digit matrices $D_{x}$ with $x \in S_{T}$ are distinct. Then, our claim follows from the observation that we can certainly choose $m=\operatorname{card}\left(S_{T}\right)$ distinct numbers $k \in \mathbb{R}$, say $\left\{k_{1}, \ldots, k_{m}\right\}$, such that the corresponding vectors $\left(\mathrm{e}^{2 \pi \mathrm{i} k_{\ell} x}\right)_{x \in S_{T}}$ with $1 \leqslant \ell \leqslant m$ are linearly independent. This gives a set of equations of the form (4) that can now be solved for the matrices $D_{x}$, with $x \in S_{T}$, as linear combinations in $B\left(k_{\ell}\right)$. Consequently, $\mathcal{B}_{D} \subseteq \mathcal{B}$ and $\mathcal{B}=\mathcal{B}_{D}$.

If the digit matrices $D_{x}$ with $x \in S_{T}$ fail to be distinct, a smaller collection of numbers $k_{\ell}$ suffices for an analogous argument.

Definition 3.3. The matrix algebra $\mathcal{B}$ of a primitive inflation rule $\varrho$ is called the inflation displacement algebra (IDA) of $\varrho$.

By construction, $\mathcal{B}$ is a subalgebra of the full matrix algebra $\operatorname{Mat}\left(n_{a}, \mathbb{C}\right)$. Recall that $\mathcal{B}$ is irreducible if the only subspaces of $\mathbb{C}^{n_{a}}$ that are invariant under the entire algebra $\mathcal{B}$ are the trivial subspaces, $\{0\}$ and $\mathbb{C}^{n_{a}}$. If there are others, $\mathcal{B}$ is called reducible. More generally, a matrix family (finite or infinite) is called irreducible if only the trivial subspaces are invariant, and reducible otherwise. ${ }^{2}$ Note that a matrix family is irreducible if and only if the algebra generated by it is. We shall later see various (classes of) examples.

Remark 3.4. The commutant $\mathcal{B}^{\prime}$ of $\mathcal{B} \subseteq \operatorname{Mat}\left(n_{a}, \mathbb{C}\right)$ is defined as

$$
\mathcal{B}^{\prime}=\left\{A \in \operatorname{Mat}\left(n_{a}, \mathbb{C}\right) \mid[A, B]=0 \text { for all } B \in \mathcal{B}\right\}
$$

and is again a subalgebra of $\operatorname{Mat}\left(n_{a}, \mathbb{C}\right)$. By Schur's lemma for the field $\mathbb{C}$, we know that $\mathcal{B}$ irreducible implies $\mathcal{B}^{\prime}=\mathbb{C} \mathbb{1}$, while the converse is generally false. If, however, our IDA $\mathcal{B}$ is closed under taking Hermitian conjugation (which turns it into a finite-dimensional $C^{*}$-algebra), von Neumann's bi-commutant theorem states that $\mathcal{B}=\mathcal{B}^{\prime \prime}=\operatorname{Mat}\left(n_{a}, \mathbb{C}\right)$, and irreducibility of $\mathcal{B}$ follows. Unfortunately, the IDA rarely is a $*$-algebra, so irreducibility has to be decided by other means. However, in view of Burnside's theorem and the fact that our alphabet has at least cardinality 2 , irreducibility in our situation is equivalent to showing that $\mathcal{B}=\operatorname{Mat}\left(n_{a}, \mathbb{C}\right) ;$ compare $[45]$ and references therein.

Remark 3.5. When a constant-length substitution $\varrho$ is bijective, meaning that every column of the word vector $\left(\varrho\left(a_{i}\right)\right)_{1 \leqslant i \leqslant n_{a}}$ is a permutation of the $n_{a}$ letters, all digit matrices $D_{x}$ are permutation matrices. This permits to compute the dimension of $\mathcal{B}=\mathcal{B}_{G}$ for some groups $G$ via the decomposition of the permutation representation $\Phi$ and some character theory.

\footnotetext{
${ }^{2}$ This notion of irreducibility is to be distinguished from the one for non-negative matrices used elsewhere in this paper. Since this will always be clear from the context, we stick to the standard terminology.
} 
In particular, when the group $G$ generated by the columns of $\varrho$ is isomorphic to the full symmetric (or permutation) group ${ }^{3} \Sigma_{n_{a}}$, one has

$$
\mathcal{B}_{\Sigma_{n_{a}}} \simeq \operatorname{Mat}\left(n_{a}-1, \mathbb{C}\right) \oplus \mathbb{C} .
$$

This follows from the fact that $\Phi$ splits as the direct sum of the trivial and the standard representation of $\Sigma_{n_{a}}$, meaning $\Phi=1 \oplus U_{\text {st }}$; compare [38].

Furthermore, when $G \simeq A_{n_{a}}$, the subgroup of even permutations, it can be shown that $\mathcal{B}_{A_{n_{a}}} \simeq \mathcal{B}_{\Sigma_{n_{a}}}$. This follows from the fact that $U_{\text {st }}$ does not split when restricted to $A_{n_{a}}$, because its character satisfies $\chi_{\text {st }}(g) \neq 0$ for some $g \in \Sigma_{n_{a}} \backslash A_{n_{a}}$, where $A_{n_{a}}$ is an index-2 subgroup of $\Sigma_{n_{a}}$; see [38, Prop. 20.13].

A subgroup for which $U_{\text {st }}$ does split is $G \simeq D_{4} \subset \Sigma_{4}$, where we have $\operatorname{dim}\left(\mathcal{B}_{D_{4}}\right)=6$; see Example 3.8 below for a substitution with such an IDA.

Before we continue, we need some result on the relation between IDAs for $\varrho$ and its powers. Assume that $\varrho$ is primitive, with substitution matrix $M_{\varrho}$ and PF eigenvalue $\lambda$. Let $B(k)$ be the Fourier matrix from Definition 3.1 for $\varrho$, and denote the corresponding matrix for $\varrho^{n}$ by $B^{(n)}(k)$, so $B^{(1)}(k)=B(k)$. A simple calculation with the displacements of $\varrho^{2}$ versus $\varrho$ shows that $B^{(2)}(k)=B(k) B(\lambda k)$ holds; we shall return to this point in more generality and detail in Section 5. Inductively, one has

$$
B^{(n+1)}(k)=B(k) B^{(n)}(\lambda k)
$$

for any $n \in \mathbb{N}$, and thus also the matrix Riesz product type relation

$$
B^{(n+1)}(k)=B(k) B(\lambda k) \cdots B\left(\lambda^{n} k\right) .
$$

Note that $B^{(n)}(k)$ defines a matrix cocycle [60] over the dilation dynamical system defined by $k \mapsto \lambda k$ on $\mathbb{R}_{+}$, which will play a central role in our later spectral analysis. We summarise the relations as follows.

Fact 3.6. Let $B(k)$ be the Fourier matrix of $\varrho$ from Definition 3.1. Then, for arbitrary $n \in \mathbb{N}$, the Fourier matrix of $\varrho^{n}$ is given by $B^{(n)}(k)=B(k) B(\lambda k) \cdots B\left(\lambda^{n-1} k\right)$.

Eq. (5) has the following consequence for the IDA of $\varrho^{n}$, denoted by $\mathcal{B}^{(n)}$.

Lemma 3.7. Let $\varrho$ be a primitive substitution over a finite alphabet with $n_{a}$ letters, and consider the corresponding inflation rule with (fixed) natural prototile lengths. If $m, n \in \mathbb{N}$ with $m \mid n$, one has $\mathcal{B}^{(n)} \subseteq \mathcal{B}^{(m)}$. In particular, $\mathcal{B}^{(n)} \subseteq \mathcal{B}^{(1)}=\mathcal{B}$ for all $n \in \mathbb{N}$.

Moreover, if there is a $q \in \mathbb{N}$ such that $\mathcal{B}^{(n)}=\operatorname{Mat}\left(n_{a}, \mathbb{C}\right)$ holds for all $n \geqslant q$, one has $\mathcal{B}^{(n)}=\operatorname{Mat}\left(n_{a}, \mathbb{C}\right)$ for all $n \in \mathbb{N}$.

Proof. Let $\lambda$ be the PF eigenvalue of $M_{\varrho}$, which implies that $\lambda^{n}$ is the corresponding one of $M_{\varrho^{n}}$. The first claim is trivial for $n=m$, so let $n>m$ and set $\ell=n / m$, which is an integer $\geqslant 2$ due to our assumptions. As a direct consequence of Eq. (5), one now derives

$$
B^{(n)}(k)=B^{(\ell m)}(k)=B^{(m)}(k) B^{(m)}\left(\lambda^{m} k\right) \cdots B^{(m)}\left(\lambda^{(\ell-1) m} k\right) .
$$

\footnotetext{
${ }^{3}$ We use $\Sigma_{n}$ for the symmetric or permutation group of $n$ symbols.
} 
This relation entails that the Fourier matrices of $\varrho^{n}$ are elements of $\mathcal{B}^{(m)}$, hence $\mathcal{B}^{(n)} \subseteq \mathcal{B}^{(m)}$ as claimed.

Now, if $\mathcal{B}^{(n)}=\operatorname{Mat}\left(n_{a}, \mathbb{C}\right)$ holds for all $n \geqslant q$, we may choose $n^{\prime}:=q$ !, so $m \mid n^{\prime}$ holds for all $1 \leqslant m \leqslant q$. The second assertion then is a consequence of the first.

The result of Lemma 3.7 looks odd at first sight, as one might expect the IDA $\mathcal{B}^{(n)}$ to be independent of $n$. However, this is generally not the case, as the next example demonstrates.

Example 3.8. Consider the alphabet $\mathcal{A}=\{a, b, c, d\}$ and the constant-length substitution $\varrho$ defined by

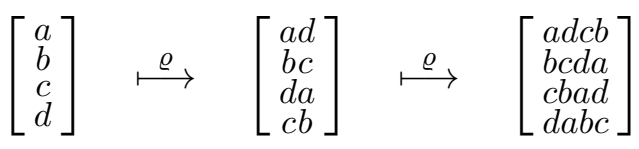

where we also wrote the second iteration. Now, in the first step, the columns display the letter permutations $(c d)$ and $(a d b c)$, which multiplicatively generate a group isomorphic with $D_{4}$. Thus, the corresponding IDA is 6-dimensional, and isomorphic with $\mathbb{C} \oplus \mathbb{C} \oplus \operatorname{Mat}(2, \mathbb{C})$. In the second step, the columns show the (non-trivial) letter permutations $(a d)(b c),(a c)(b d)$ and $(a b)(c d)$, which only generate Klein's 4-group, $C_{2} \times C_{2}$, which is Abelian. Here, the IDA is then 4-dimensional, and isomorphic to $\mathbb{C} \oplus \mathbb{C} \oplus \mathbb{C} \oplus \mathbb{C}$. More generally, $\mathcal{B}^{(2 n)}=\mathcal{B}^{(2)}$ and $\mathcal{B}^{(2 n+1)}=\mathcal{B}^{(1)}$ for all $n \in \mathbb{N}$.

We say that $\varrho$ admits a substitutional root if a substitution $\sigma$ exists such that $\varrho=\sigma^{n}$ for some $n \geqslant 2$. A necessary condition for this to happen is that the corresponding substitution matrices satisfy $M_{\varrho}=M_{\sigma}^{n}$. This condition is not sufficient, as one can see from $\varrho=(a b a, a b)$, which has the square root $(a b, a)$, versus $\varrho^{\prime}=(a a b, a b)$, which has no root. Nevertheless, $M_{\varrho}=M_{\varrho^{\prime}}$, and the two substitutions even generate the same hull. The following consequence of Lemma 3.7 is immediate.

Corollary 3.9. Let @ be a primitive inflation rule with irreducible IDA. If $\sigma$ is a substitutional root of $\varrho$, the IDA of $\sigma$, when realised with the matching tile lengths, is irreducible as well.

Let us next state one general sufficient criterion for the irreducibility of an IDA.

Proposition 3.10. Let $\varrho$ be a primitive substitution over a finite alphabet with $n_{a} \geqslant 2$ letters. If the natural prototile lengths are distinct, the IDA of $\varrho$ is $\mathcal{B}=\operatorname{Mat}\left(n_{a}, \mathbb{C}\right)$ and hence irreducible.

Proof. If $\varrho$ is primitive, we know that the hull defined by it is minimal, and each element of it is linearly repetitive; see [5] and references therein for background. In particular, there is a number $\zeta>0$ such that every (connected) legal patch of length $\geqslant \zeta$ contains at least one copy of each prototile. Denote the natural prototile lengths by $\ell_{1}, \ldots, \ell_{n_{a}}$, where we may assume that the letters of the alphabet are ordered such that $\ell_{1}>\ell_{2}>\ldots>\ell_{n_{a}}>0$.

Define $\triangle_{\min }=\min \left\{\ell_{1}-\ell_{2}, \ell_{2}-\ell_{3}, \ldots, \ell_{n_{a}}-\ell_{n_{a}-1}\right\}$. Then, we pick an integer $q$ such that $\lambda^{q} \triangle_{\min }>\zeta$, with $\lambda$ the PF eigenvalue of $M_{\varrho}$ as before, and consider $\varrho^{q}$. This power of $\varrho$ now has the property that the corresponding level-1 supertile of type $i$, which is the patch $\varrho^{q}\left(a_{i}\right)$, 
is longer than that of type $i+1$ by more than $\zeta$, and this holds for all $1 \leqslant i \leqslant n_{a}-1$. If we draw the level-1 supertiles in a stack on top of each other, with coinciding left endpoints, we see that each supertile now has an 'overhang' of length $>\zeta$ over the next one below it. We can now determine the digit matrices $D_{x}^{(q)}$ of $\varrho^{q}$ as follows.

For each $1 \leqslant i \leqslant n_{a}$, by our above repetitivity argument, at least one $x \in T_{i, 1}^{(q)}$ exists with $x>\lambda^{q} \ell_{2}$, and the corresponding digit matrix is $D_{x}^{(q)}=E_{i, 1}$, the standard elementary matrix. Consequently, all $E_{i, 1}$ are in $\mathcal{B}^{(q)}$. Next, for each $1 \leqslant i \leqslant n_{a}$, there exists at least one $x \in T_{i, 2}^{(q)}$ with $x>\lambda^{q} \ell_{3}$, and we have $\left(D_{x}^{(q)}\right)_{i, 2}=1$. Here, we do not know whether the first column of $D_{x}^{(q)}$ contains only zeros, as there could be some coincidences between $\varrho\left(a_{1}\right)$ and $\varrho\left(a_{2}\right)$. However, if $\left(D_{x}^{(q)}\right)_{j, 1}=1$ for some $j$, we may form differences with the elementary matrix $E_{j, 1}$, which we already know to be in $\mathcal{B}^{(q)}$. So, also all $E_{i, 2}$ are in $\mathcal{B}^{(q)}$.

Proceeding inductively in the row number, we see (after finitely many steps) that all matrices $E_{i, j}$ must be in $\mathcal{B}^{(q)}$, wherefore we get $\mathcal{B}^{(q)}=\operatorname{Mat}\left(n_{a}, \mathbb{C}\right)$. Since our argument with the sufficiently long overhangs applies to all powers $\varrho^{q^{\prime}}$ with $q^{\prime} \geqslant q$, the second assertion of Lemma 3.7 implies that $\mathcal{B}=\operatorname{Mat}\left(n_{a}, \mathbb{C}\right)$ as well, which proves the main claim.

Since we are working over $\mathbb{C}^{n_{a}}$ with $n_{a} \geqslant 2$, irreducibility follows from Burnside's theorem, as $\mathbb{C}$ is algebraically closed; see $[41,45]$.

In view of Eq. (4) and the ensuing discussion of $\mathcal{B}$ versus $\mathcal{B}_{D}$, the following is immediate.

Corollary 3.11. Let @ be a primitive substitution over a finite alphabet with irreducible IDA $\mathcal{B}$. Then, for each $\varepsilon>0$, the complex algebra generated by the matrices $\{B(k) \mid 0 \leqslant k<\varepsilon\}$ is again $\mathcal{B}$, and hence irreducible as well. Moreover, even the finite matrix family $\{B(k) \mid k \in J\}$ is irreducible, provided that $J$ contains at least $r=\left|S_{T}\right|$ distinct values of $k$ that are rationally independent.

The point here is that, if $\mathcal{B}$ is irreducible, no matrix family $\{B(k) \mid 0 \leqslant k<\varepsilon\}$ with $\varepsilon>0$ can possess a non-trivial invariant subspace. This can be viewed as a first step towards establishing a stronger irreducibility notion, as needed for a version of Furstenberg's theorem to represent extremal Lyapunov exponents; compare [60, Ch. 6] and [23, Thm. 2.3].

Note that the IDA of a primitive inflation rule $\varrho$ is not an MLD invariant, see [5, Sec. 5.2] for background, and neither is its irreducibility. Since the latter is an important tool, we illustrate this phenomenon with a paradigmatic example.

Example 3.12. The classic Thue-Morse (TM) rule

$$
\varrho_{\mathrm{TM}}: \quad 1 \mapsto 1 \overline{1}, \quad \overline{1} \mapsto \overline{1} 1
$$

defines a substitution of constant length over the binary alphabet $\{1, \overline{1}\}$, which can thus also be read as an inflation rule for two prototiles of unit length; compare [5, Secs. 4.6 and 10.1]. It possesses a (self-explanatory) bar swap symmetry in the sense of [3], which implies the IDA 
$\mathcal{B}_{\mathrm{TM}}$ to be reducible. In fact,

$$
\mathcal{B}_{\mathrm{TM}}=\left\{\left(\begin{array}{ll}
\alpha & \beta \\
\beta & \alpha
\end{array}\right) \mid \alpha, \beta \in \mathbb{C}\right\}
$$

is a two-dimensional commutative subalgebra of $\operatorname{Mat}(2, \mathbb{C})$; see $[3$, Sec. 4.1$]$ for details.

Next, observe that any sequence in the TM hull is composed of overlapping words of the form $1 \overline{1}^{\ell} 1$ with $\ell \in\{0,1,2\}$, where the overlap with the preceding (ensuing) word is always 1 . These are nothing but the three right-collared return words for the letter 1 of the TM system; see [25] for background. Consequently, each 1 in the sequence is the first letter of one of the right-collared words (in obvious notation)

$$
a=\left.1 \overline{1} \overline{1}\right|_{1}, \quad b=\left.1 \overline{1}\right|_{1} \quad \text { or } \quad c=\left.1\right|_{1},
$$

so that there is a simple rule between the TM hull and the derived $a b c$ hull with natural tile lengths, which is local in both directions. This makes the two hulls MLD as tiling spaces, where $a, b$ and $c$ represent prototiles of lengths 3,2 and 1, respectively.

Now, it is easy to check that we inherit an inflation rule for the new prototiles, namely

$$
\varrho^{\prime}: \quad a \mapsto a b c, \quad b \mapsto a c, \quad c \mapsto b,
$$

which satisfies the conditions of Proposition 3.10, and thus possesses Mat $(3, \mathbb{C})$ as its IDA, which is irreducible. Note that, for the inflation $\varrho^{\prime}$, the positions (left endpoints) of the tiles $a, b$ and $c$ in any fixed element of the hull taken together coincide with the positions of all intervals of type 1 in the corresponding element of the original TM hull.

Example 3.13. Closely related is the period doubling substitution $\varrho_{\mathrm{pd}}=(A B, A A)$ on the alphabet $\{A, B\}$. It defines a subshift that is a factor of the TM system, with a globally $2: 1$ factor map [5, Thm. 4.7]. In the notation of Example 3.12, the latter is given by the sliding block map defined via $\psi(1 \overline{1})=\psi(\overline{1} 1)=A$ and $\psi(11)=\psi(\overline{1} \overline{1})=B$. It is not difficult to check that the IDA of $\varrho_{\mathrm{pd}}$ is generated by the digit matrices

$$
D_{0}=\left(\begin{array}{ll}
1 & 1 \\
0 & 0
\end{array}\right) \quad \text { and } \quad D_{1}=\left(\begin{array}{ll}
0 & 1 \\
1 & 0
\end{array}\right) .
$$

Since $D_{0} D_{1}=D_{0}$ and $D_{1} D_{0}=\left(\begin{array}{ll}0 & 0 \\ 1 & 1\end{array}\right)$, one sees that $\mathcal{B}_{\mathrm{pd}}$ is a three-dimensional algebra, namely

$$
\mathcal{B}_{\mathrm{pd}}=\left\{\left(\begin{array}{cc}
\alpha & \alpha+\gamma \\
\beta+\gamma & \beta
\end{array}\right) \mid \alpha, \beta, \gamma \in \mathbb{C}\right\},
$$

which is non-commutative, but still reducible, with non-trivial invariant subspace $\mathbb{C}\left(\begin{array}{c}1 \\ -1\end{array}\right)$.

As in our previous example, we can use the return words

$$
a=\left.A\right|_{A} \quad \text { and } \quad b=\left.A B\right|_{A}
$$

to construct an inflation rule with distinct tile lengths that defines an MLD system, then with an irreducible IDA. 
Remark 3.14. Examples 3.12 and 3.13 can be extended to general constant-length substitutions over a binary alphabet $\mathcal{A}=\{a, b\}$ as follows. If $\varrho=\left(w_{a}, w_{b}\right)$, with $w_{a}$ and $w_{b}$ being words of the same length, is primitive and aperiodic, the corresponding IDA is either $\mathcal{B}_{\mathrm{pd}}$ or $\mathcal{B}_{\mathrm{TM}}$. The latter case occurs if and only if $\varrho$ is bijective (meaning that $w_{a}$ and $w_{b}$ differ at every position). Nevertheless, in all these examples, the induced inflation rule for the new alphabet based on the return words has an irreducible IDA, but defines a tiling system that is MLD with the previous one.

The structure of the IDA becomes more complex, and more interesting, for larger alphabets and in higher dimensions. Here, we discuss one example, and return to IDAs later, in Section 4 and in Example 5.12.

Remark 3.15. For the Rudin-Shapiro substitution [51, 5], as defined by $\varrho_{\mathrm{RS}}=(02,32,01,31)$ on $\mathcal{A}=\{0,1,2,3\}$, a return word encoding does not lead to tiles of distinct length. Indeed, the eight right-collared return words for the letter 0 are

$$
\left.01\right|_{0},\left.02\right|_{0},\left.0131\right|_{0},\left.0232\right|_{0},\left.013132\right|_{0},\left.01313231\right|_{0},\left.02323132\right|_{0},\left.0232313231\right|_{0},
$$

and using them to set up the new alphabet $\mathcal{A}^{\prime}=\{a, b, c, \ldots, h\}$, in the same order, one gets the induced substitution $\varrho_{\mathrm{RS}}^{\prime}=(d, b a, g, b c a, h a, h e, b c f a, b c f e)$, which is again primitive. Its substitution matrix is

$$
M=\left(\begin{array}{llllllll}
0 & 1 & 0 & 1 & 1 & 0 & 1 & 0 \\
0 & 1 & 0 & 1 & 0 & 0 & 1 & 1 \\
0 & 0 & 0 & 1 & 0 & 0 & 1 & 1 \\
1 & 0 & 0 & 0 & 0 & 0 & 0 & 0 \\
0 & 0 & 0 & 0 & 0 & 1 & 0 & 1 \\
0 & 0 & 0 & 0 & 0 & 0 & 1 & 1 \\
0 & 0 & 1 & 0 & 0 & 0 & 0 & 0 \\
0 & 0 & 0 & 0 & 1 & 1 & 0 & 0
\end{array}\right)
$$

with eigenvalues $2, \pm \sqrt{2},-1$ and 0 (the last with multiplicity 4 ). The PF left eigenvector is $(2,2,4,4,6,8,8,10)$ for the natural interval lengths, with length 2 for the shortest interval to reflect the meaning of $a$ in the original version, while the corresponding right eigenvector $\frac{1}{16}(4,4,2,2,1,1,1,1)^{T}$ codes the letter frequencies as usual.

It is interesting to note that, in the geometric realisation with natural interval lengths, we thus have a system that is not of constant length, but shows absolutely continuous diffraction (and thus also spectral) components. This follows from the fact that the Rudin-Shapiro system and this geometric return word system are MLD, hence lead to topologically conjugate dynamical systems under the translation action of $\mathbb{R}$.

The IDA of the induced inflation rule is irreducible, which has an interesting consequence on the way the AC spectrum is encoded in the Fourier matrix cocycle. In particular, we do no longer have a $k$-independent subspace with unitary dynamics as in the original version $[3,46]$, but a $k$-dependent equivariant family. This would deserve further exploration, in particular via extending some results in this direction from [23]. 


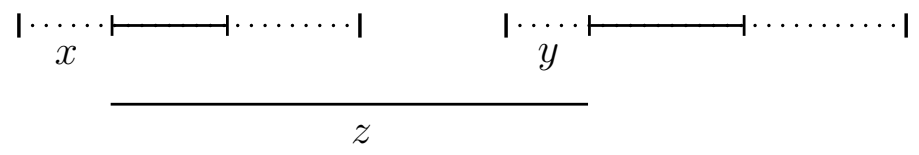

Figure 1. If two tiles (solid intervals) at distance $z$ have offsets $x$ and $y$ within their covering supertiles (dotted intervals), the latter have distance $z+x-y$.

For the appropriate treatment of pair correlations, one has to go one step beyond the IDA in considering the real algebra generated by the matrices $\boldsymbol{A}(k)=B(k) \otimes \overline{B(k)}$. Since we need rather little of this extension below, we summarise the basic properties in an appendix.

3.2. Pair correlation functions and measures. Since the structure of the correlation measures for periodic examples is clear, we can restrict our attention to primitive inflation rules that are aperiodic in the sense of [5, Def. 4.13]. Note that, in one dimension, the hull of a primitive inflation rule is either periodic or aperiodic. Let $\Lambda=\Lambda_{1} \cup \cdots \cup \Lambda_{n_{a}}$ be a fixed point of the aperiodic, primitive inflation rule $\varrho$ (or of $\varrho^{q}$ for some $q \in \mathbb{N}$, which defines the same hull), where each interval of type $a_{i}$ carries a marker point of type $i$ at its left endpoint. As in [3], we define $\nu_{i j}(z)$ as the relative frequency of the occurrence of distance $z$ between a point of type $i$ (left) and one of type $j$ (right). These quantities exist uniformly due to unique ergodicity, and they are constant on the hull due to minimality. In fact, for any $\Lambda$ from the hull, one has the same relation,

$$
\nu_{i j}(z)=\frac{\operatorname{dens}\left(\Lambda_{i} \cap\left(\Lambda_{j}-z\right)\right)}{\operatorname{dens}(\Lambda)},
$$

which entails $\nu_{i j}(0)=0$ for $i \neq j$ and $\sum_{i=1}^{n_{a}} \nu_{i i}(0)=1$.

Clearly, we also have

$$
\nu_{i j}(-z)=\nu_{j i}(z)
$$

for all $i, j$ and all $z$. Moreover, we know that

$$
\nu_{i j}(z)>0 \Longleftrightarrow \nu_{i j}(z) \neq 0 \quad \Longleftrightarrow \quad z \in S_{i j}:=\Lambda_{j}-\Lambda_{i},
$$

where the Minkowski differences $\Lambda_{j}-\Lambda_{i}$ are again the same for each element of the hull, whence $S_{i j}$ is well defined. Note that the first equivalence is clear by definition. One direction of the second equivalence follows from the geometric constraint of the tiling, while the other is another consequence of minimality (and hence repetitivity).

In [3], the pair correlation functions $\nu_{i j}(z)$ for the example of the Fibonacci chain are shown to satisfy a set of exact, linear renormalisation equations, which we now extend to primitive inflation rules in full generality as follows.

Lemma 3.16. Let $\mathbb{Y}$ be the tiling space defined by an aperiodic, primitive inflation rule $\varrho$, with a fixed set of $n_{a}$ prototiles of natural length. Given some $\Lambda \in \mathbb{Y}$, let $\nu_{i j}(z)$ be the relative frequency of occurrence of a tile of type $i$ (left) and one of type $j$ (right) at distance 
$z$ between their left endpoints, which exists and is independent of $\Lambda$. Then, these coefficients are non-negative and satisfy the renormalisation equations

$$
\nu_{m n}(z)=\frac{1}{\lambda} \sum_{i, j=1}^{n_{a}} \sum_{x \in T_{m i}} \sum_{y \in T_{n j}} \nu_{i j}\left(\frac{z+x-y}{\lambda}\right),
$$

for $1 \leqslant m, n \leqslant n_{a}$, together with the symmetry relation (7) and the support condition (8).

Sketch of proof. The derivation works in complete analogy to the Fibonacci case in [3], employing local recognisability, as illustrated in Figure 1. In fact, it is not necessary that we start from a fixed point, because the pair correlation functions exist, and are the same for every element of the hull $\Lambda$, which is minimal. The derivation only requires the recognition of the unique level-1 supertile to which any individual tile belongs.

Remark 3.17. The derivation of Eq. (9) relies on the unique identification of the covering supertile for each tile, but it does not require this process to be local. Therefore, the statement of Lemma 3.16 can also be extended to primitive inflation rules that define a periodic hull. In such a case, one consistently marks the supertiles in each element of the hull, for which one has more than one choice, and proceeds with the otherwise unchanged proof. It is not difficult to check that the outcome does not depend on the actual decomposition chosen. $\diamond$

For some aspects, as exploited in [3], we need to understand precisely to what extent the renormalisation equations (9) determine the frequencies. Let us thus look at these relations from scratch, for which we first need to recall some results from the theory of matrices with non-negative entries.

A matrix $M \in \operatorname{Mat}(d, \mathbb{Z})$, written as $M=\left(m_{i j}\right)_{1 \leqslant i, j \leqslant d}$, is called non-negative if $m_{i j} \geqslant 0$ for all $i, j$. We assume the reader to be familiar with the classic notions of irreducibility and primitivity of such matrices, and with the classic theorems due to Perron and Frobenius; compare [5, Sec. 2.4] and references given there, or [34, Ch. 13] for a detailed account. Moreover, we shall need some other properties that are best stated via the normal form, $M_{\mathrm{nf}}$, of a non-negative matrix $M$. Following [34, Ch. 13.4], it is given by

$$
M_{\mathrm{nf}}=\left(\begin{array}{ccc|ccc}
M_{1} & & \mathbf{0} & & & \\
& \ddots & & & \mathbf{0} & \\
\mathbf{0} & & M_{r} & & & \\
\hline M_{r+1,1} & \cdots & M_{r+1, r} & M_{r+1} & & \mathbf{0} \\
\vdots & & & \ddots & \ddots & \\
M_{s, 1} & & \cdots & & M_{s, s-1} & M_{s}
\end{array}\right)
$$

where $s \geqslant r \geqslant 1$ and all $M_{i}$ are indecomposable, ${ }^{4}$ non-negative square matrices, and where, if $s>r$, each sequence $M_{r+\ell, 1}, \ldots, M_{r+\ell, r+\ell-1}$ contains at least one non-zero matrix. Such a

\footnotetext{
${ }^{4}$ A square matrix $M$ is called decomposable if it can be brought to the block-triangular form $M^{\prime}=\left(\begin{array}{ll}A & 0 \\ B\end{array}\right)$ via simultaneous permutations of its rows and columns, and indecomposable otherwise [34].
} 
normal form can always be achieved by a suitable permutation of the coordinates, hence by the corresponding simultaneous permutation of the rows and columns of $M$. It is essentially unique, up to obvious permutations of entire blocks, which clearly do not change the values of $r$ and $s$.

In particular, we need the following result, which is a simple extension of [34, Thm. 13.7].

Lemma 3.18. Consider a non-negative matrix $M$ in normal form $M_{\mathrm{nf}}$ according to Eq. (10), and let $\lambda$ be an eigenvalue of $M$. Then, $M$ has a corresponding strictly positive eigenvector, meaning that all entries are positive, if and only if

(1) $M_{i}$ has eigenvalue $\lambda$ for every $1 \leqslant i \leqslant r$;

(2) No $M_{j}$ with $r<j \leqslant s$ has $\lambda$ as an eigenvalue.

In this situation, one has $\lambda>0$, and the eigenspace of $\lambda$ is one-dimensional if and only if $r=1$.

Having exact renormalisation relations for the pair correlation functions in Lemma 3.16, where the support of these functions also follows from the inflation construction, it is a natural question to what extent these relations, taken on their own, determine the $\nu_{i j}$. This is tantamount to asking what the solution space of (9) is, and how it depends on the prescribed support of the $\nu_{i j}$. The latter point is critical, due to the occurrence of scaled arguments, and increasing the support might increase the solution space; compare [3].

Theorem 3.19. Assume that the geometric data needed to write down Eq. (9) are taken from $a$ (periodic or aperiodic) primitive inflation rule @ with inflation multiplier $\lambda$ as explained above. Let $\nu_{i j}$, with $1 \leqslant i, j \leqslant n_{a}$, be real-valued functions with $\operatorname{supp}\left(\nu_{i j}\right) \subseteq \Delta_{i j} \subset \mathbb{R}$, where the $\Delta_{i j}$ are given point sets such that $\Delta:=\bigcup_{i, j} \Delta_{i j}$ is locally finite and contains 0 . Then, the solution space of the linear renormalisation equations (9) with functions of this type is finite-dimensional. When $S_{i j} \subseteq \Delta_{i j}$ for all $1 \leqslant i, j \leqslant n_{a}$, this solution space is non-trivial.

In particular, when $\Delta_{i j}=S_{i j}$ for all $1 \leqslant i, j \leqslant n_{a}$, the solution space is one-dimensional, so there is precisely one solution with $\sum_{i=1}^{n_{a}} \nu_{i i}(0)=1$, which is actually strictly positive. This solution automatically satisfies the symmetry relation (7). If $\Delta_{i j}$ is further restricted to a true subset of $S_{i j}$ for at least one index pair, the solution space becomes trivial.

Proof. Observe first that, since $\lambda>1$, the set of equations with

$$
|z| \leqslant c:=\frac{1}{\lambda-1} \sup \left\{x-y \mid x \in T_{m i}, y \in T_{n j}, 1 \leqslant i, j, m, n \leqslant n_{a}\right\}
$$

forms a closed subsystem of linear equations. When the total support is inside a fixed locally finite set $\Delta \subset \mathbb{R}$, we know that $\Delta \cap[-c, c]$ is a finite set, wherefore the closed subsystem comprises finitely many equations only (there is at least one, since $0 \in \Delta$ by assumption).

Observe next that we only need to prove the dimensionality claim for this subsystem. Indeed, since $\Delta \subset \mathbb{R}$ is locally finite, there is a smallest $z \in \Delta$ with $z>c$, and the values of the correlation coefficients at $z$ are then uniquely determined, either from the support constraint or from Eq. (9), where only arguments with modulus $<z$ (and hence $\leqslant c$ in this case) occur on the right-hand side. Proceeding inductively with growing modulus of $z$, one 
sees that the correlation coefficients are uniquely determined for all $z>c$. An analogous argument works for all $z \in \Delta$ with $z<-c$.

Consequently, the solution space dimension of the entire system, with functions $\nu_{i j}$ with $\operatorname{supp}\left(\nu_{i j}\right) \subseteq \Delta$, equals that of the closed, finite subsystem. This proves the first claim, while the underlying inflation tiling space with its properties guarantees at least one solution if the supports are large enough; compare Eq. (8).

Indeed, when $\Delta_{i j}=S_{i j}=\Lambda_{j}-\Lambda_{i}$, we know from Eq. (8) that a strictly positive solution exists, with $\operatorname{supp}\left(\nu_{i j}\right)=\Delta_{i j}$ for all $1 \leqslant i, j \leqslant n_{a}$. Consider the subset of equations of (9) that emerge from inserting $z=0$ on the left-hand side. Since $\Lambda_{i} \cap \Lambda_{j}=\varnothing$ for $i \neq j$, one has $0 \in S_{i j}$ if and only if $i=j$. This means that we must have $\nu_{i j}(0)=0$ for all $i \neq j$. Moreover, if $x \in T_{m i}$ and $y \in T_{n j}$, one has $0 \leqslant x<\lambda \ell_{i}$ and $0 \leqslant y<\lambda \ell_{j}$ and hence the inequality

$$
-\ell_{j}<\frac{x-y}{\lambda}<\ell_{i}
$$

from the geometry of the prototiles. So, for $x \neq y$, the point $\frac{x-y}{\lambda}$ cannot be an element of $S_{i j}$, and $\nu_{i j}$ must vanish there. We thus remain with the relations

$$
\nu_{m m}(0)=\frac{1}{\lambda} \sum_{i=1}^{n_{a}} \operatorname{card}\left(T_{m i}\right) \nu_{i i}(0),
$$

for $1 \leqslant m \leqslant n_{a}$, which together give the eigenvalue equation

$$
M_{\varrho}\left(\begin{array}{c}
\nu_{11}(0) \\
\vdots \\
\nu_{n_{a} n_{a}}(0)
\end{array}\right)=\lambda\left(\begin{array}{c}
\nu_{11}(0) \\
\vdots \\
\nu_{n_{a} n_{a}}(0)
\end{array}\right) .
$$

By primitivity of $\varrho$, the non-negative matrix $M_{\varrho}$ is primitive, and the eigenspace of $\lambda=\lambda_{\mathrm{PF}}$ is one-dimensional. Moreover, there is an eigenvector with $\nu_{i i}(0)>0$ for all $i$, which can be normalised as $\sum_{i=1}^{n_{a}} \nu_{i i}(0)=1$. If we now multiply both sides of Eq. (9) by $\lambda$, we may interpret it as an eigenvector equation for eigenvalue $\lambda$, where we have finitely many vector components of the form $\nu_{i j}(z)$ with $z \in S_{i j}$ and $|z| \leqslant c$ from above. The right-hand side of Eq. (9) can now be seen as the application of a non-negative matrix $A$ to this vector, where $A$ is decomposable, because we have identified an irreducible subblock in Eq. (11).

Since we know from the underlying inflation $\varrho$ together with Eq. (8) that $A$ has $\lambda$ as an eigenvalue with a strictly positive eigenvector corresponding to it, we deduce from Lemma 3.18 that the eigenspace for $\lambda$ is one-dimensional if no other invariant diagonal subblock of $A$ exists with eigenvalue $\lambda$, meaning $r=1$ in Lemma 3.18.

Indeed, when there is some $0<z \in S_{i j}$, we know that there exists a legal patch of the form $a_{i} w a_{j}$, with $w$ a finite word and $a_{i} w$ coding a patch of length $z$. From the structure of the primitive inflation $\varrho$, we know that $a_{i} w a_{j}$ must then be a subword of $\varrho^{k}\left(a_{1}\right)$ for some $k \in \mathbb{N}$, where we may assume $k$ to be the minimal such power. Now, applying the renormalisation equation $k$ times to $a_{1}$ implies that $\nu_{i j}(z)$ is linked to $\nu_{11}(0)$ on this level, as the patch under consideration lies in this very level- $k$ supertile. Consequently, $\nu_{i j}(z)$ cannot belong to a decoupling subset of components. An analogous argument holds for negative $z$. 
We thus see that the parameter $r$ of the normal form $A_{\text {nf }}$ of $A$ must be $r=1$, and the dimension of our solution space is indeed 1 when $\Delta_{i j}=S_{i j}$. The symmetry is clear, while the final claim is now a simple consequence of Eq. (8), which followed from the strict ergodicity of our underlying dynamical system.

Remark 3.20. One can rewrite Eq. (9) in a slightly different way that establishes a link to the generators of the $\mathbb{R}$-algebra $\mathcal{A}$ from the Appendix, as originally studied in [2, Sec. 5.2] in the case of binary alphabets. If we use $\underline{\nu}=\left(\nu_{11}, \nu_{12}, \ldots, \nu_{n_{a} n_{a}}\right)^{T}$ in the standard ordering for a double index, one can check that Eq. (9) can be rewritten as

$$
\lambda \underline{\nu}(z)=\sum_{x \in S_{T}-S_{T}} F_{x} \underline{\nu}\left(\frac{z+x}{\lambda}\right),
$$

where we use (as before) the convention to set $\nu_{i j}(z)=0$ whenever $z$ is not in the admissible set $\Delta_{i j}$. Note that this version of the renormalisation equation can be read as an eigenvector equation for a non-negative matrix.

3.3. Renormalisation for correlation measures and their Fourier transforms. Let us return to the hull $\mathbb{Y}$ defined by the primitive inflation rule $\varrho$, and consider some $\Lambda \in \mathbb{Y}$. The Minkowski difference $\Delta=\Lambda-\Lambda$ is locally finite and the same set for all elements $\Lambda \in \mathbb{Y}$. We may thus consistently define pure point measures $\Upsilon_{m n}=\sum_{z \in \Lambda-\Lambda} \nu_{m n}(z) \delta_{z}$ as in [3], called the pair correlation measures. Now, Eqs. (7) and (8) imply

$$
\widetilde{\Upsilon_{m n}}=\Upsilon_{n m} \text { and } \Upsilon_{m n} \geqslant 0
$$

Moreover, each $\Upsilon_{m m}$ is a positive definite measure, and, due to Eq. (6), see also [2, Eq. 4.1], one actually has the representation

$$
\Upsilon_{m n}=\frac{\widetilde{\delta_{\Lambda_{m}}} \circledast \delta_{\Lambda_{n}}}{\operatorname{dens}(\Lambda)}
$$

which could also be used to define the measures in the first place.

With this definition, Eq. (9) implies the measure-valued counterpart

$$
\Upsilon_{m n}=\frac{1}{\lambda} \sum_{i, j=1}^{n_{a}} \sum_{r \in T_{m i}} \sum_{s \in T_{n j}} \delta_{s-r} *\left(f . \Upsilon_{i j}\right),
$$

as originally derived in [2, Lemma 4.2] for the binary case. Here, $f(x):=\lambda x$ and $f \cdot \mu$ is defined via $(f . \mu)(\mathcal{E})=\mu\left(f^{-1}(\mathcal{E})\right)$ for Borel sets $\mathcal{E}$, which matches with the definition via test functions used earlier. Note that the $\Upsilon_{i j}$ are the pair correlation measures both of the entire hull $\mathbb{Y}$ and of each individual member of $\mathbb{Y}$.

Remark 3.21. The pair correlation measures can be considered as the building blocks of diffraction theory as follows. Decompose $\Lambda \in \mathbb{Y}$ as $\Lambda=\dot{\bigcup}_{1 \leqslant i \leqslant n_{a}} \Lambda_{i}$ into the distinct types of points, and consider the measure $\omega=\sum_{i=1}^{n_{a}} u_{i} \delta_{\Lambda_{i}}$ with weights $u_{i} \in \mathbb{C}$, which is translation 
bounded by construction. Its autocorrelation $\gamma=\gamma_{u}$ according to Eq. (1) exists, and reads

$$
\gamma_{u}=\operatorname{dens}(\Lambda) \sum_{i, j=1}^{n_{a}} \overline{u_{i}} \Upsilon_{i j} u_{j}
$$

which also implies that one has $\widehat{\gamma_{u}}=\operatorname{dens}(\Lambda) \sum_{i, j} \bar{u}_{i} \widehat{\Upsilon}_{i j} u_{j}$. In this sense, understanding $\Upsilon$ and $\widehat{\Upsilon}$ gives complete access to the diffraction measures of the dynamical system.

Observe that, by Lemma 2.1, all measures $\Upsilon_{m n}$ are translation bounded and transformable. As a result of Eq. (13), we also know that

$$
\overline{\widehat{\Upsilon_{m n}}}=\widehat{\widehat{\Upsilon_{m n}}}=\widehat{\Upsilon_{n m}},
$$

and each $\widehat{\Upsilon_{m n}}$ is a positive definite measure, with $\widehat{\Upsilon_{m m}} \geqslant 0$ in addition for all $m$.

Recalling from [8, Lemma 2.5] that $\widehat{f . \mu}=\frac{1}{\lambda}\left(f^{-1} \cdot \widehat{\mu}\right)$ holds for any transformable measure $\mu$, and applying Fourier transform and the convolution theorem to (15), one finds

$$
\widehat{\Upsilon_{m n}}=\frac{1}{\lambda^{2}} \sum_{i, j=1}^{n_{a}} \sum_{r \in T_{m i}} \sum_{s \in T_{n j}} \mathrm{e}^{-2 \pi \mathrm{i}(s-r)(.)}\left(f^{-1} \cdot \widehat{\Upsilon_{i j}}\right),
$$

which is the appropriate generalisation of the Fibonacci equations from [3] to this more general situation. Let us rewrite Eq. (17) in matrix form to highlight its structure. Employing column vector notation for the Kronecker product structure discussed in Remark 3.20 and in the Appendix, with

$$
\Upsilon:=\left(\Upsilon_{11}, \Upsilon_{12}, \ldots, \Upsilon_{1 n_{a}}, \Upsilon_{21}, \Upsilon_{22}, \ldots, \Upsilon_{n_{a} n_{a}}\right)^{T}
$$

and similarly for the Fourier transform, one now finds

$$
\widehat{\Upsilon}=\frac{1}{\lambda^{2}} \boldsymbol{A}(.)\left(f^{-1} . \widehat{\Upsilon}\right)
$$

with $\boldsymbol{A}(k)=B(k) \otimes \overline{B(k)}$ in complete analogy to the Fibonacci example treated in [3], and $B(k)$ as in Definition 3.1; see also [2, Prop. 4.3]. Several properties of the matrices $\boldsymbol{A}(k)$ and the algebra generated by them are collected in the Appendix.

Next, observe that each component $\widehat{\Upsilon_{i j}}$ has a unique decomposition into its pure point (pp) and continuous (c) parts, where the support of the pure point part is (at most) a countable set. The union of these countable sets over $i, j$ still is a countable set, and justifies the decomposition of the measure vector $\widehat{\Upsilon}$ as $\widehat{\Upsilon}=(\widehat{\Upsilon})_{\mathrm{pp}}+(\widehat{\Upsilon})_{\mathrm{c}}$. This means that we have a decomposition $\mathbb{R}=\mathcal{E}_{\mathrm{pp}} \cup \dot{\mathcal{E}} \mathcal{E}_{\mathrm{c}}$ such that

$$
(\widehat{\Upsilon})_{\mathrm{pp}}=\left.\widehat{\Upsilon}\right|_{\mathcal{E}_{\mathrm{pp}}} \text { and }(\widehat{\Upsilon})_{\mathrm{c}}=\left.\widehat{\Upsilon}\right|_{\mathcal{E}_{\mathrm{c}}}
$$

where $\mathcal{E}_{\mathrm{pp}}$ is a countable set. Without loss of generality, we may also assume that $\mathcal{E}_{\mathrm{pp}}=f\left(\mathcal{E}_{\mathrm{pp}}\right)$, for instance by replacing $\mathcal{E}_{\mathrm{pp}}$ with $\mathcal{E}_{\mathrm{pp}}^{\prime}:=\bigcup_{m \in \mathbb{Z}} f^{m}\left(\mathcal{E}_{\mathrm{pp}}\right)$, which is still a countable set, and $\mathcal{E}_{\mathrm{c}}$ with $\mathcal{E}_{\mathrm{c}}^{\prime}:=\mathbb{R} \backslash \mathcal{E}_{\mathrm{pp}}^{\prime}$, which is then also invariant under $f$. This gives another valid decomposition of $\mathbb{R}$, which is better suited for our purposes. 
Similarly, one can now further split the continuous component into its singular continuous (sc) and absolutely continuous (ac) parts, finally giving

$$
\widehat{\Upsilon}=(\widehat{\Upsilon})_{\mathrm{pp}}+(\widehat{\Upsilon})_{\mathrm{sc}}+(\widehat{\Upsilon})_{\mathrm{ac}},
$$

where each part is concentrated on a set that is a null set for the other two parts. Note that we may assume, without loss of generality, that the supporting sets are disjoint and invariant under the linear mapping $f$. This follows constructively by an extension of our previous argument to a decomposition of $\mathcal{E}_{\mathrm{c}}^{\prime}$ into two sets, leading to $\mathbb{R}=\mathcal{E}_{\mathrm{pp}}^{\prime} \dot{\cup} \mathcal{E}_{\mathrm{sc}}^{\prime} \dot{\cup} \mathcal{E}_{\mathrm{ac}}^{\prime}$ with $(\widehat{\Upsilon})_{\alpha}=\left.\widehat{\Upsilon}\right|_{\mathcal{E}_{\alpha}^{\prime}}$ and $f\left(\mathcal{E}_{\alpha}^{\prime}\right)=\mathcal{E}_{\alpha}^{\prime}$ for all $\alpha \in\{\mathrm{pp}, \mathrm{sc}, \mathrm{ac}\}$.

Lemma 3.22. The scaling relation (18) for $\widehat{\Upsilon}$ holds for each of the three spectral types separately. In other words, one has

$$
(\widehat{\Upsilon})_{\alpha}=\frac{1}{\lambda^{2}} \boldsymbol{A}(.)\left(f^{-1} \cdot \widehat{\Upsilon}\right)_{\alpha}
$$

for each $\alpha \in\{\mathrm{pp}, \mathrm{sc}, \mathrm{ac}\}$. Moreover, Eq. (16) holds for each spectral type separately.

Proof. Note that the matrix function $\boldsymbol{A}(k)$ depends analytically on $k$, wherefore the measure vectors $\boldsymbol{A}(.) \boldsymbol{\mu}$ and $\boldsymbol{\mu}$ are of the same spectral type; in particular, $(\boldsymbol{A}(.) \boldsymbol{\mu})_{\alpha}=\boldsymbol{A}(.)(\boldsymbol{\mu})_{\alpha}$ holds for each $\alpha \in\{\mathrm{pp}, \mathrm{sc}, \mathrm{ac}\}$. Moreover, the map $\boldsymbol{\mu} \mapsto f^{-1} \cdot \boldsymbol{\mu}$, which is a simple dilation, does not change the spectral type either. The claim is now an exercise in restricting the measures on the left-hand and right-hand sides to the $f$-invariant supporting sets $\mathcal{E}_{\alpha}^{\prime}$ together with their measure-theoretic orthogonality; compare [5, Prop. 8.4].

The last claim follows from standard arguments.

3.4. Analysis of pure point part. Let us first take a closer look at the pure point part, which can be written as

$$
(\widehat{\Upsilon})_{\mathrm{pp}}=\operatorname{dens}(\Lambda) \sum_{k \in \mathcal{E}_{\mathrm{pp}}^{\prime}} \boldsymbol{I}(k) \delta_{k}
$$

with the (at most countable) set $\mathcal{E}_{\mathrm{pp}}^{\prime}$ introduced earlier. Here, the extra factor $\operatorname{dens}(\Lambda)$ is introduced to match the definition of $\boldsymbol{I}(k)$ as a relative (or dimensionless) quantity with the interpretation of $\widehat{\gamma_{u}}(0)$ according to Remark 3.21 and [5, Prop. 9.2]. As before, we use a vector notation, with the intensity vector $\boldsymbol{I}$, so $\left(\widehat{\Upsilon_{i j}}\right)_{\mathrm{pp}}=\operatorname{dens}(\Lambda) \sum_{k \in \mathcal{E}_{\mathrm{pp}}^{\prime}} I_{i j}(k) \delta_{k}$. As a result of Eq. (16) and Lemma 3.22, we have $I_{i i}(k) \geqslant 0$ together with

$$
\overline{I_{i j}(k)}=I_{j i}(k)=I_{i j}(-k) .
$$

A straight-forward calculation with point measures in the form of weighted Dirac combs, in conjunction with a comparison of coefficients, now shows that $\boldsymbol{I}$ must also satisfy the identity

$$
\boldsymbol{I}(k)=\lambda^{-2} \boldsymbol{A}(k) \boldsymbol{I}(\lambda k)
$$

for all $k$, with the obvious understanding that we set $I_{i j}(k)=0$ for any $k$ outside the supporting set $\mathcal{E}_{\mathrm{pp}}^{\prime}$. In particular, for $k=0$, Eq. (19) entails the relation

$$
\boldsymbol{A}(0) \boldsymbol{I}(0)=\lambda^{2} \boldsymbol{I}(0) \text {. }
$$


Since $\boldsymbol{A}(0)=M_{\varrho} \otimes M_{\varrho}$, which has PF eigenvalue $\lambda^{2}$, we recognise this as an eigenvalue equation that is related to the frequencies of the prototiles, respectively the density of the subsets of points of the corresponding type. In fact, the solution is unique up to an overall constant, and given by

$$
I_{i j}(0)=\alpha^{2} \operatorname{dens}\left(\Lambda_{i}\right) \operatorname{dens}\left(\Lambda_{j}\right),
$$

where $\alpha=\operatorname{dens}(\Lambda)^{-1}$ in our setting due to the definition of the $\nu_{i j}(z)$ as relative (and hence dimensionless) frequencies. Note that this contribution, which decouples from other values of $k$, is always present, no matter whether our system has non-trivial point spectrum or not.

Let us briefly look at the above scaling relations in a different way. Defining the matrix $\mathcal{I}=\left(I_{i j}\right)_{1 \leqslant i, j \leqslant n_{a}}$, one checks that Eq. (19) can be rewritten as

$$
\mathcal{I}(k)=\lambda^{-2} B(k) \mathcal{I}(\lambda k) B(k)^{\dagger},
$$

where $^{\dagger}$ denotes Hermitian conjugation. Clearly, this implies the relation

$$
|\operatorname{det}(B(k))|^{2} \operatorname{det}(\mathcal{I}(\lambda k))=\lambda^{2 n_{a}} \operatorname{det}(\mathcal{I}(k)),
$$

which has various consequences. In particular, $\operatorname{det}(\mathcal{I}(k))$ will usually vanish. Moreover, the matrix $\mathcal{I}(0)$ has rank 1, as follows from Eq. (21). In fact, a more general result is true.

Theorem 3.23. Let $\varrho$ be a primitive inflation rule with hull $\mathbb{Y}$. Then, for all $k \in \mathbb{R}$, there are numbers $a_{i}(k)$, with $1 \leqslant i \leqslant d$, such that the identity $I_{i j}(k)=\overline{a_{i}(k)} a_{j}(k)$ holds for $1 \leqslant i, j \leqslant n_{a}$. These numbers are the dimensionless Fourier-Bohr coefficients, defined as

$$
a_{j}(k)=\alpha \lim _{r \rightarrow \infty} \frac{1}{2 r} \sum_{x \in \Lambda_{j} \cap[-r+c, r+c]} \mathrm{e}^{-2 \pi \mathrm{i} k x},
$$

with $\alpha=\operatorname{dens}(\Lambda)^{-1}$. Here, the convergence is uniform in $c \in \mathbb{R}$. This coefficient is independent of the choice of $\Lambda \in \mathbb{Y}$. As a consequence, for all $k \in \mathbb{R}$, the matrix $\mathcal{I}(k)$ is Hermitian, positive semi-definite, and has rank at most 1 . Moreover, the set of $k$ with $a_{j}(k) \neq 0$ for some $j$ is at most a countable set.

Proof. Our system is strictly ergodic, and all eigenfunctions are continuous [51]. This implies the uniform existence of the Fourier-Bohr coefficients [37, 43], as well as their independence of the choice of $\Lambda$.

For $I_{i i}(k)$, the claim is now a consequence of [43, Thm. 5] applied to $\delta_{\Lambda_{i}}$, which gives $I_{i i}(k)=\left|a_{i}(k)\right|^{2}$, where $I_{i i}(k)>0$ at most for countably many $k \in \mathbb{R}$. For $I_{i j}(k)$ with $i \neq j$, we recall Eqs. (2) and (14) to obtain

$$
\Upsilon_{i j}=\frac{1}{4} \sum_{\ell=1}^{4} \mathrm{i}^{\ell}\left[\left(\omega_{j}+\mathrm{i}^{\ell} \omega_{i}\right) \circledast\left(\omega_{j}+\mathrm{i}^{\ell} \omega_{i}\right)^{\sim}\right]
$$

with $\omega_{i}=\delta_{\Lambda_{i}}$. Since the contribution of the term in square brackets to $I_{i j}(k)$ is given by $\left|a_{j}(k)+\mathrm{i}^{\ell} a_{i}(k)\right|^{2}$, again by [43, Thm. 5], one finds $I_{i j}(k)=\overline{a_{i}(k)} a_{j}(k)$ as claimed. 
Remark 3.24. The result of Theorem 3.23 can be viewed as a variant of the Bombieri-Taylor observation on the connection between exponential sums (or amplitudes) and intensities. Its original version refers to the relations $I_{i i}(k)=\left|a_{i}(k)\right|^{2}$. For primitive inflation rules, they extend to all components of $\mathcal{I}(k)$ as stated.

Remark 3.25. The representation of $\mathcal{I}(k)$ as a rank-1 matrix in Theorem 3.23 is only unique up to a phase, which means that replacing $a(k)$ by $\mathrm{e}^{-2 \pi \mathrm{i} \vartheta(k)} a(k)$ results in the same $\mathcal{I}(k)$. When $\Lambda, \Lambda^{\prime} \in \mathbb{Y}$ are translates of one another, say $\Lambda^{\prime}=t+\Lambda$, one has $\vartheta(k)=k t$, but more complicated phase functions show up in general, due to the structure of $\mathbb{Y}$. This is an interesting problem in its own right, and has been studied extensively in the physics literature; see $[59$, Sec. 4.2$]$ and references therein.

Still, our recursion has the consequence that we also have

$$
\left|a_{i}(k)\right|=\lambda^{-1}\left|\sum_{j} B_{i j}(k) \overline{a_{j}(\lambda k)}\right|,
$$

which can be further analysed when $B(k)$ is invertible. It can provide valuable insight on how the modulus of the amplitudes behaves under the inward or outward iteration.

3.5. Analysis of absolutely continuous part. Let us now take a closer look at $(\widehat{\Upsilon})_{\mathrm{ac}}$. By the Radon-Nikodym theorem, each component $\left(\widehat{\Upsilon_{i j}}\right)_{\text {ac }}$ is represented by a measurable and locally integrable density function $h_{i j}$ relative to Lebesgue measure, though this is generally not an element of $L^{1}(\mathbb{R})$.

For the next result, we consider $W=\mathbb{C}^{n_{a}} \otimes \mathbb{C}^{n_{a}}$ also as a real vector space, then of dimension $2 n_{a}^{2}$, and split it as $W=W_{+} \oplus W_{-}$into the eigenspaces of the $\mathbb{R}$-linear map $C$ on $W$ defined by $x \otimes y \mapsto \bar{y} \otimes \bar{x}$, where : is complex conjugation; see the Appendix for more.

Lemma 3.26. Let $\boldsymbol{h}$ be the vector of Radon-Nikodym densities that represents $\widehat{\Upsilon}_{\mathrm{ac}}$. Then, one has the relation

$$
\boldsymbol{h}(k)=\frac{1}{\lambda} \boldsymbol{A}(k) \boldsymbol{h}(\lambda k),
$$

which holds for Lebesgue-a.e. $k \in \mathbb{R}$.

Moreover, the relations $h_{i j}(-k)=h_{j i}(k)=\overline{h_{i j}(k)}$ and $h_{i i}(k) \geqslant 0$ hold for a.e. $k \in \mathbb{R}$ and all $1 \leqslant i, j \leqslant n_{a}$. In particular, $\boldsymbol{h}(k) \in W_{+}$for a.e. $k \in \mathbb{R}$.

Proof. Let $g$ be a continuous function with compact support in $\mathbb{R}$. We have to determine what the relation from Lemma 3.22, for $\alpha=$ ac, implies for the Radon-Nikodym densities of $\boldsymbol{\mu}:=\widehat{\Upsilon}_{\mathrm{ac}}$. The left-hand side of Lemma 3.22 clearly reads

$$
\boldsymbol{\mu}(g)=\int_{\mathbb{R}} g(k) \mathrm{d} \boldsymbol{\mu}(k)=\int_{\mathbb{R}} g(k) \boldsymbol{h}(k) \mathrm{d} k .
$$

With $f(k)=\lambda k$ as before, we can calculate the right-hand side of Lemma 3.22 as follows,

$$
\begin{aligned}
\lambda^{-2}\left(\boldsymbol{A}(.)\left(f^{-1} \cdot \boldsymbol{\mu}\right)\right)(g) & =\lambda^{-2} \int_{\mathbb{R}} g\left(f^{-1}(k)\right) \boldsymbol{A}\left(f^{-1}(k)\right) \mathrm{d} \boldsymbol{\mu}(k) \\
& =\lambda^{-2} \int_{\mathbb{R}} g\left(\frac{k}{\lambda}\right) \boldsymbol{A}\left(\frac{k}{\lambda}\right) \boldsymbol{h}(k) \mathrm{d} k=\frac{1}{\lambda} \int_{\mathbb{R}} g(k) \boldsymbol{A}(k) \boldsymbol{h}(\lambda k) \mathrm{d} k,
\end{aligned}
$$


where the first step is just working out the definition of the measure on the left, while the last is the result of a change of variable transformation. This is the important step, as it leads to the cancellation of one factor of $\lambda$ in the denominator. Comparing the two expressions above, and observing that the test function $g$ was arbitrary, leads to the first claim by means of standard arguments for densities.

The second claim is a consequence of Eq. (16) and Lemma 3.22 for the absolutely continuous parts, in conjunction with the properties of the correlation measures $\Upsilon_{i j}$ from Eq. (13). Since the action of the mapping $C$ on $\boldsymbol{h}$ is given by $(C \boldsymbol{h})_{i j}(k)=\overline{h_{j i}(k)}$, the previous claim implies $(C \boldsymbol{h})(k)=\boldsymbol{h}(k)$ for a.e. $k \in \mathbb{R}$, hence $\boldsymbol{h}(k) \in W_{+}$as claimed.

To continue, we need the following well-known decomposition property of positive semidefinite, Hermitian matrices, which we prove for convenience.

Fact 3.27. Let $H=\left(h_{i j}\right)_{1 \leqslant i, j \leqslant d} \in \operatorname{Mat}(d, \mathbb{C})$ be Hermitian and positive semi-definite, with rank $m$. Then, all diagonal elements of $H$ are non-negative. If $h_{i i}=0$ for some $i$, one has $h_{i j}=h_{j i}=0$ for all $1 \leqslant j \leqslant d$.

Whenever $H \neq 0$, there are $m \geqslant 1$ Hermitian, positive semi-definite matrices $H_{1}, \ldots, H_{m}$ of rank 1 such that $H=\sum_{r=1}^{m} H_{r}$ together with $H_{r} H_{s}=0$ for $r \neq s$.

Proof. By Sylvester's criterion, $H$ positive semi-definite means that all principal minors are non-negative, hence in particular all diagonal elements of $H$. Assume $h_{i i}=0$ for some $i$, and select any $j \in\{1, \ldots, d\}$. By semi-definiteness in conjunction with Hermiticity, one finds

$$
0=h_{i i} h_{j j} \geqslant h_{i j} h_{j i}=\left|h_{i j}\right|^{2} \geqslant 0,
$$

which implies the second claim.

Employing Dirac's notation, the spectral theorem for Hermitian matrices asserts that one has $H=\sum_{i=1}^{d}\left|v_{i}\right\rangle \lambda_{i}\left\langle v_{i}\right|$, where the eigenvectors $\left|v_{i}\right\rangle$ can be chosen to form an orthonormal basis (so $\left\langle v_{i} \mid v_{j}\right\rangle=\delta_{i, j}$ and $\left|v_{i}\right\rangle\left\langle v_{i}\right|$ is a projector of rank 1), while all eigenvalues are nonnegative due to positive semi-definiteness. The rank of $H$ is the number of positive eigenvalues, counted with multiplicities. Ordering the eigenvalues decreasingly as $\lambda_{1} \geqslant \lambda_{2} \geqslant \cdots \geqslant \lambda_{d} \geqslant 0$, one can choose $H_{r}=\left|v_{r}\right\rangle \lambda_{r}\left\langle v_{r}\right|$ for $1 \leqslant r \leqslant m$, and the claim is obvious.

In order to profit from Lemma 3.26, we now perform a dimensional reduction as follows. ${ }^{5}$ Define the matrix $\mathcal{H}(k)=\left(h_{i j}(k)\right)_{1 \leqslant i, j \leqslant n_{a}}$, which is Hermitian and positive semi-definite, for a.e. $k \in \mathbb{R}$. Simply switching from a notation with vectors of length $n_{a}^{2}$ to a version with $n_{a} \times n_{a}$ matrices, the renormalisation relation can be rewritten as

$$
\mathcal{H}(k)=\lambda^{-1} B(k) \mathcal{H}(\lambda k) B^{\dagger}(k) .
$$

\footnotetext{
${ }^{5}$ As we outline in more detail in the Appendix, one can alternatively work with the recursion from Lemma 3.26 directly. However, the dimensional reduction leads to a stronger result in the sense that we also get a representation of $\boldsymbol{h}$ that resembles the situation of the pure point part.
} 
If we decompose $\mathcal{H}(k)=\sum_{i=1}^{m} \mathcal{H}_{i}(k)$ as a sum of Hermitian, positive semi-definite matrices of rank 1 according to Fact 3.27, every term is of the form

$$
\mathcal{H}_{i}(k)=v^{(i)}(k)\left(v^{(i)}\right)^{\dagger}(k)
$$

where each $v^{(i)}(k)$ is a vector of functions from $L_{\text {loc }}^{2}(\mathbb{R})$. Now, one has

$$
B(k) \mathcal{H}(k) B^{\dagger}(k)=\sum_{i=1}^{m} B(k) \mathcal{H}_{i}(k) B^{\dagger}(k),
$$

and we can study the simpler iteration

$$
v(k)=\frac{1}{\sqrt{\lambda}} B(k) v(\lambda k)
$$

instead of (22). Iterating this relation, for a.e. $k \in \mathbb{R}$, yields

$$
v\left(\frac{k}{\lambda^{n}}\right)=\frac{1}{\lambda^{n / 2}} B\left(\frac{k}{\lambda^{n-1}}\right) \cdots B\left(\frac{k}{\lambda}\right) v(k),
$$

which we call the inward iteration for $v(k)$, with given $k \in \mathbb{R}$. When $B(k)$ is invertible, at least for a.e. $k \in \mathbb{R}$, we get

$$
v(\lambda k)=\sqrt{\lambda} B^{-1}(k) v(k)
$$

and the corresponding outward iteration,

$$
v\left(\lambda^{n} k\right)=\lambda^{n / 2} B^{-1}\left(\lambda^{n-1} k\right) \cdots B^{-1}(\lambda k) B^{-1}(k) v(k),
$$

which holds for a.e. $k \in \mathbb{R}$ and is of particular interest to us. For the existence of the matrix inverses on a set of full measure, it suffices that $\operatorname{det}(B(k)) \neq 0$ for some $k \in \mathbb{R}$, because the determinant is an analytic function in $k$ and thus can then at most have isolated zeros.

This way, we can consider the corresponding matrix cocycle, whose Lyapunov exponents determine the asymptotic growth behaviour of $v$ for $k \rightarrow \infty$. It is sufficient to look at the extremal ones, which are derived from the $B^{(n)}(k)$ previously defined in Eq. (5) and Fact 3.6. Writing out $B^{(n)}(k)$ and its inverse, these extremal exponents are given by

$$
\begin{aligned}
& \chi_{\max }(k)=\log \sqrt{\lambda}+\limsup _{n \rightarrow \infty} \frac{1}{n} \log \left\|B^{-1}\left(\lambda^{n-1} k\right) \cdots B^{-1}(k)\right\| \\
& \chi_{\min }(k)=\log \sqrt{\lambda}+\liminf _{n \rightarrow \infty} \frac{1}{n} \log \left\|B(k) B(\lambda k) \cdots B\left(\lambda^{n-1} k\right)\right\|^{-1} ;
\end{aligned}
$$

compare [60, Eq. 2.2]. This follows from the general definition, compare [60], applied to the iteration from (25) by a simple calculation, where the additional logarithmic term reflects the multiplication by $\sqrt{\lambda}$ in each iteration step. Now, one also obtains

$$
\chi_{\min }(k)=\log \sqrt{\lambda}-\chi^{B}(k)
$$

where

$$
\chi^{B}(k):=\limsup _{n \rightarrow \infty} \frac{1}{n} \log \left\|B^{(n)}(k)\right\|
$$

is the maximal Lyapunov exponent of the Fourier matrix cocycle, $B^{(n)}(k)$. This way, we can formulate one of our central results in terms of the asymptotic behaviour of the Fourier matrices of $\varrho^{n}$ for large $n$ as follows, which is the expected extension of the result for a binary 
example from [2] to general alphabets, but requires a slightly different proof. Also, we state it in terms of the Fourier matrix cocycle, $B^{(n)}(k)$, as this seems the most natural object.

Theorem 3.28. Let $\varrho$ be a primitive inflation rule, with inflation multiplier $\lambda$, and let $B(k)$ be the corresponding Fourier matrix, with $\operatorname{det}(B(k)) \neq 0$ for some $k \in \mathbb{R}$. If there is an $\varepsilon>0$ such that $\chi^{B}(k) \leqslant \log \sqrt{\lambda}-\varepsilon$ holds for a.e. $k \in \mathbb{R}$, where $\chi^{B}(k)$ is the maximal Lyapunov exponent of the Fourier matrix cocycle, the diffraction measure of the system cannot have an absolutely continuous part.

Proof. If such an $\varepsilon>0$ exists, there is a $0<\delta \leqslant \varepsilon$ such that $\left\|v\left(\lambda^{n} k\right)\right\|$, for a.e. $k$, is bounded below by $C \mathrm{e}^{\delta n}$ as $n \rightarrow \infty$, where the constant can depend on $k$, but is always positive, so this lower bound grows exponentially. This allows an application of [2, Lemma 9.3]: Translation boundedness of the diffraction measure means $\int_{0}^{a \lambda^{m}}\|v(x)\|_{2}^{2} \mathrm{~d} x=\mathcal{O}\left(a \lambda^{m}\right)$ for any $a>0$ as $m \rightarrow \infty$, while a positive exponent means that there is some $\eta>1$ with $\int_{0}^{a \lambda^{m}}\|v(x)\|_{2}^{2} \mathrm{~d} x \geqslant c_{v}(\eta \lambda)^{m}$. These conflicting conditions are compatible only when $\|v\|_{2}^{2}=0$, so $v(k)=0$ for a.e. $k \in \mathbb{R}$.

For any choice of the weight vector $u \in \mathbb{C}^{n_{a}}$, the absolutely continuous part of the corresponding diffraction measure, $\widehat{\gamma}_{a c}$, is a translation-bounded measure. If $u_{j}=\delta_{j, \ell}$ for some fixed index $\ell$, the Radon-Nikodym density of $\widehat{\gamma}_{a c}$ is the locally integrable function $h_{\ell \ell} \geqslant 0$. Since a finite sum of translation-bounded measures is still translation bounded, $h_{s}:=\sum_{\ell=1}^{n_{a}} h_{\ell \ell}$ represents a translation-bounded, positive measure.

Now, by Fact 3.27 in conjunction with Eqs. (22) and (25), there is an integer $m \leqslant n_{a}$ such that $h_{s}$ is of the form

$$
h_{s}(k)=\sum_{\ell=1}^{n_{a}} \sum_{i=1}^{m}\left|v_{\ell}^{(i)}(k)\right|^{2} .
$$

Since each summand is non-negative, there can be no cancellation between the terms, and the exponential growth of any of them would violate translation boundedness as explained above. Consequently, we must have $v^{(i)}(k)=0$ for a.e. $k \in \mathbb{R}$ and for all $1 \leqslant i \leqslant m$, and hence $\widehat{\Upsilon}_{\mathrm{ac}}=0$. This implies $\widehat{\gamma}_{\mathrm{ac}}=0$ as claimed.

In fact, as shown in [2], $\chi_{\min }(k)>0$ for a subset of full measure of some interval of the form $\left[\frac{\varepsilon}{\lambda}, \varepsilon\right]$ with $\varepsilon>0$ is already enough to rule out an absolutely continuous diffraction component. This leads to the following consequence.

Corollary 3.29. If the primitive inflation rule $\varrho$, with inflation multiplier $\lambda$ and the determinant condition on $B(k)$ as before, leads to a system that displays a non-trivial diffraction component of absolutely continuous type, one must have $\chi_{\min }(k) \leqslant 0$ on a subset of $\mathbb{R}$ of positive measure. When $\chi_{\min }(k)$ is constant for a.e. $k \in \mathbb{R}$, this constant must be $\leqslant 0$.

This means that we are in a particularly good situation whenever Oseledec's theorem applies, see [60, Ch. 4] for backgropund, as is the case for constant-length substitutions.

Remark 3.30. In [19], a variant of the cocycle $B^{n}(k)$ for $S$-adic systems is considered, called the spectral cocycle. There, the main objects are spectral measures $\sigma_{f}$ of a certain class of 
functions associated to a suspension flow. One of the main results is the expression of the lower local dimension $\underline{d}\left(\sigma_{f}, x\right)$ in terms of the Lyapunov exponent of the spectral cocycle. It is shown that the singularity of these measures is implied by the Lyapunov exponent being strictly bounded from above by $\log \sqrt{\lambda_{\boldsymbol{a}}}$ for a.e. $k \in \mathbb{R}^{d}$, see [19, Cor. 2.4], where $\lambda_{\boldsymbol{a}}$ is the generalisation of the $\mathrm{PF}$ eigenvalue for $S$-adic systems (which is actually also defined as a Lyapunov exponent).

Flows generated by deterministic substitutions form a subclass of $S$-adic systems, for which a dynamical version of the singularity result in Theorem. 3.28 for almost every choice of roof function is shown in [19, Cor. 2.6]. The one-dimensional systems we cover in this work pertain to the specific case where the roof function is given by the left PF eigenvector of the substitution matrix of $\varrho$.

3.6. Further consequences. In theory, the Lyapunov exponent could still be negative, thus signifying an exponential decay in the outward direction. However, since the Radon-Nikodym densities $\boldsymbol{h}(k)$ can be recovered from the vectors $v(k)$, one can rule out the existence of negative exponents via measure-theoretic arguments when the elements $\Lambda$ of the geometric hull $\mathbb{Y}$ satisfy some additional properties.

Proposition 3.31. Assume that the elements of $\mathbb{Y}$ are Meyer sets, and assume that $\widehat{\gamma}_{\mathrm{ac}} \neq 0$. Then, its Radon-Nikodym density cannot decay at infinity.

Proof. When the point set $\Lambda \in \mathbb{Y}$ under consideration is a Meyer set, it was recently shown by Strungaru [58] that the absolutely continuous part of its diffraction measure is of the form $\widehat{\gamma}_{\mathrm{ac}}=\widehat{\mu}$ where $\mu$ is a pure point measure with Meyer set support. Moreover, it follows from [35, Cor. 11.1] that $\widehat{\mu}$ is a strongly almost periodic measure. Consequently, for any $g \in C_{\mathrm{c}}(\mathbb{R})$, the convolution $f:=\widehat{\mu} * g$ is a Bohr almost periodic function. By starting from a non-negative $g$ with sufficiently small support, we make sure that $f \neq 0$.

If the Radon-Nikodym density $h$ of $\widehat{\mu}$ decays at infinity, then so does $\widehat{\mu}$ as a measure, and hence also $f$. On the other hand, for any $\varepsilon>0$, the $\varepsilon$-almost periods of $f$ are relatively dense. This only leaves $f=0$, which is a contradiction, and $h$ cannot decay at infinity.

For primitive inflation rules that lead to a hull $\mathbb{Y}$ of Meyer sets with non-trivial absolutely continuous diffraction, we can neither have $\chi_{\min }<0$, by Proposition 3.31 , nor $\chi_{\min }>0$, by Corollary 3.29, which has the following rather strong consequence. For its formulation, let $\chi^{B}(v, k):=\limsup _{n \rightarrow \infty} \frac{1}{n} \log \left\|B^{(n)}(k) v\right\|$.

Corollary 3.32. Assume that the hull $\mathbb{Y}$ contains Meyer sets only, and that the diffraction measure $\widehat{\gamma}_{\mathrm{ac}}$ is nontrivial. Then, $\chi^{B}(v, k)=\log \sqrt{\lambda}$ holds for a set of $k \in \mathbb{R}$ of positive measure, for all $v=v^{(i)}(k)$ as in $\mathcal{H}_{i}$ from Eq. (23). In particular, this criterion applies whenever the inflation multiplier $\lambda$ of the primitive substitution is a PV number. Whenever $\chi^{B}(v, k)$ is constant a.e., this constant must be $\log \sqrt{\lambda}$.

Proof. While the first claim is clear from the previous arguments, the second follows from the fact that the geometric realisation of a primitive Pisot (or PV) substitution always leads to a geometric hull with the Meyer property. 
Let us be more precise with the last point. Though we still do not know whether the Pisot substitution conjecture holds, we do know that the fixed point of a primitive PV inflation is a relatively dense subset of a model set, and hence a Meyer set; see [55] and references therein for the details. Since every hull of a primitive PV inflation is minimal and can be generated as the orbit closure of a fixed point (possibly under some power of the inflation rule), compare [5, Sec. 4.2], the corresponding hull consists of Meyer sets only.

The last claim of the corollary is clear.

In favourable situations, some of which will be discussed in the next section, one can calculate the Lyapunov exponents explicitly, and then apply Theorem 3.28 directly. Otherwise, one can look for upper bounds to $\chi^{B}(k)$ in order to establish the estimate needed in Theorem 3.28. Let us briefly explain one particularly useful method that is based on a subadditivity argument; compare [28].

Since the norm in Eq. (26) is arbitrary, we may choose a submultiplicative one, such as the spectral norm or the Frobenius norm. Then, for any $m, n \in \mathbb{N}$, one has

$$
\left\|B^{(m+n)}(k)\right\|=\left\|B^{(m)}(k) B^{(n)}\left(\lambda^{m} k\right)\right\| \leqslant\left\|B^{(m)}(k)\right\|\left\|B^{(n)}\left(\lambda^{m} k\right)\right\|
$$

and thus, with $L_{n}(k):=\log \left\|B^{(n)}(k)\right\|$, also

$$
L_{m+n}(k) \leqslant L_{m}(k)+L_{n}\left(\lambda^{m} k\right) .
$$

Using arithmetic progressions, which correspond to averages along sequences that are totally Bohr ergodic in the sense of [28], one can invoke a standard argument known from Fekete's subadditive lemma. Indeed, for a fixed $N \in \mathbb{N}$, one has

$$
\frac{L_{q N+r}(k)}{q N+r} \leqslant \frac{q}{q N+r}\left(\frac{1}{q} \sum_{\ell=0}^{q-1} L_{N}\left(\lambda^{\ell N} k\right)\right)+\frac{L_{r}\left(\lambda^{q N} k\right)}{q N+r},
$$

where $q \in \mathbb{N}$ and $0 \leqslant r<N$. As $q \rightarrow \infty$, under mild conditions that are satisfied in our case, the first term with the Birkhoff sum converges to the mean, namely $\frac{1}{N} \mathbb{M}\left(L_{N}\right)$ as detailed below, while the second converges to zero, both for a.e. $k \in \mathbb{R}$. One thus obtains the following bound for the maximal Lyapunov exponent of $B^{(n)}$. For further details of the proof, we refer to $[2$, Lemma 6.16] and the treatment in [9].

Lemma 3.33. Let $B^{(n)}($.$) be the Fourier matrix cocycle of a primitive inflation rule with$ inflation multiplier $\lambda>1$, and let $L_{n}(k)=\log \left\|B^{(n)}(k)\right\|$, for every $n \in \mathbb{N}$, be Bohr almost periodic. Then, for any $N \in \mathbb{N}$ and a.e. $k \in \mathbb{R}$, one has

$$
\chi^{B}(k)=\limsup _{n \rightarrow \infty} \frac{1}{n} L_{n}(k) \leqslant \frac{1}{N} \mathbb{M}\left(L_{N}\right),
$$

where $\mathbb{M}(f):=\lim _{T \rightarrow \infty} \frac{1}{T} \int_{0}^{T} f(t) \mathrm{d} t$ is the mean of the function $f$.

Note that the mean of an almost periodic function always exists, and that Bohr almost periodicity can be relaxed by an application of Sobol's theorem; compare [9]. In the more restrictive situation that $\lambda$ is an integer and $L_{N}$ is thus 1-periodic, one can determine the 
mean via Birkhoff's ergodic theorem [26, Thm. 2.30] whenever $L_{N}$ is integrable in the Lebesgue sense, as explained in some detail in [9, Sec. 6.3].

In general, observing that $L_{N}$ is a quasiperiodic function, its mean can be evaluated as an integral over the $D$-torus, where $D$ is the algebraic degree of $\lambda$. Indeed, each matrix entry $B_{i j}(k)$ is quasiperiodic, and can thus be represented as a section through a function $P_{i j}(\tilde{k})$ that is 1-periodic in each of the (possibly several) variables; compare [8, Secs. 3.1 and 5.2] for details. This works simutaneously for the entire matrix $B(k)$, and analogously for $B^{(N)}(k)$. Employing the Frobenius norm, one then has

$$
\frac{1}{N} \mathbb{M}\left(\log \left\|B^{(N)}(.)\right\|_{\mathrm{F}}^{2}\right)=\frac{1}{N} \int_{\mathbb{T}^{D}} \log \left(\sum_{i, j=1}^{n_{a}}\left|P_{i j}^{(N)}(\tilde{k})\right|^{2}\right) \mathrm{d} \tilde{k}
$$

where the 1-periodic trigonometric polynomials $P_{i j}^{(N)}$ are the entries of $B^{(N)}$. Effectively, one can now calculate the right-hand side of the upper bound from Lemma 3.33 for increasing $N$, and test this against the threshold value of $\log \sqrt{\lambda}$ from Theorem 3.28. It turns out that this works quite well in concrete examples; see $[8,7]$ and Sections 4 and 5.

It is reasonable to expect that $\chi^{B}(v, k) \leqslant \log \sqrt{\lambda}$ for a.e. $k \in \mathbb{R}$ holds under more general circumstances, that is, possibly for all $v$, and also beyond the Meyer set case.

Theorem 3.34. Let $\varrho$ be a primitive inflation rule, with multiplier $\lambda$ and Fourier matrix $B(k)$. Then, for a.e. $k \in \mathbb{R}$, one has $\chi_{\min }(k) \geqslant 0$ or, equivalently, that $\chi^{B}(k) \leqslant \log \sqrt{\lambda}$.

Proof. Choosing the Frobenius norm for convenience, the mean of $\log \left\|B^{(N)}\right\|_{\mathrm{F}}$ can be calculated as explained above around Eq. (27). Now, via Jensen's inequality, we get

$$
\exp \left(\mathbb{M}\left(\log \left\|B^{(N)}(.)\right\|_{\mathrm{F}}^{2}\right)\right) \leqslant \int_{\mathbb{T}^{D}} \sum_{i, j=1}^{n_{a}}\left|P_{i j}^{(N)}(\tilde{k})\right|^{2} \mathrm{~d} \tilde{k}=\sum_{i, j}\left\|P_{i j}^{(N)}\right\|_{2}^{2}=\sum_{i, j}\left(M^{N}\right)_{i j}
$$

with $M$ being the substitution matrix of $\varrho$. The last step follows from Parseval's equation and the observation that the coefficients of the trigonometric polynomials can only be 0 or 1 , due to the nature of the control points (see below for more), and $B^{(N)}(0)=M^{N}$.

Now, since $\varrho$ is primitive, we know that $\left(M^{N}\right)_{i j} \sim C \lambda^{N} v_{i} u_{j}$ holds simultaneously for all $i, j$ as $N \rightarrow \infty$, where $C>0$ is some constant, and $u$ and $v$ are the left and right PF eigenvectors of $M$, both strictly positive and conveniently normalised (via $\sum_{i} v_{i}=\sum_{i} u_{i} v_{i}=1$, say). In fact, the error term of this estimate is exponentially small because all other eigenvalues of $M$ are strictly smaller than $\lambda$ in modulus. This gives

$$
\frac{1}{N} \mathbb{M}\left(\log \left\|B^{(N)}(.)\right\|_{\mathrm{F}}^{2}\right) \leqslant \frac{1}{N} \log \left(C^{\prime} \lambda^{N}\right)=\log (\lambda)+\frac{1}{N} \log \left(C^{\prime}\right)
$$

for some $C^{\prime}>0$ and all sufficiently large $N$. Consequently, we also have

$$
\chi^{B}(k) \leqslant \liminf _{N \rightarrow \infty} \frac{1}{2 N} \mathbb{M}\left(\log \left\|B^{(N)}(.)\right\|_{\mathrm{F}}^{2}\right) \leqslant \frac{1}{2} \log (\lambda)=\log \sqrt{\lambda}
$$

from Lemma 3.33 for a.e. $k \in \mathbb{R}$ as claimed. 
The previous theorem is a strong almost everywhere result that has to be satisfied by generic primitive substitutions. This, together with Theorem 3.28, provides a necessary criterion for the presence of AC spectral components as follows.

Corollary 3.35. Let $\varrho$ be a primitive inflation rule, with multiplier $\lambda$ and Fourier matrix $B(k)$, where we assume that $\operatorname{det}(B(k)) \neq 0$ for some $k$. Let $B^{(n)}(k)$ be the Fourier matrix cocycle as before. If the diffraction measure of the hull defined by @ comprises a non-trivial absolutely continuous component, one has $\chi^{B}(k)=\log \sqrt{\lambda}$ for a subset of $\mathbb{R}$ of positive measure, or for a.e. $k \in \mathbb{R}$ whenever $\chi^{B}(k)$ is almost surely constant.

To date, few examples are known where a non-trivial AC component occurs at all, namely the Rudin-Shapiro sequence [51, 5] and its relatives, which all have a similar structure [29]. To the best of our knowledge, no example outside the class of constant-length substitutions (and their topological conjugates, which need not be of constant length) is known.

Let us briefly compare Corollary 3.35 with another necessary criterion, taken from [17], for constant-length substitutions, which reads as follows.

Theorem 3.36 ([17, Thm. 1.1]). Let @ be a primitive substitution of constant length, and let $M$ be its substitution matrix, with PF eigenvalue $\lambda$. If $\widehat{\gamma}_{\mathrm{ac}} \neq 0$, there is an eigenvalue $\alpha$ of $M$ with $|\alpha|=\sqrt{\lambda}$.

In hindsight, this criterion is a restriction on the Lyapunov exponents for the inward iteration from Eq. (24). What we have in Corollary 3.35, under the non-degeneracy assumption for $\operatorname{det}(B(k))$, is a condition on the outward iteration - hence a criterion that applies to primitive inflations in general. Taken together, this suggests even more that absolutely continuous components are only possible under very restrictive conditions.

\section{Abelian Bijective substitutions}

It is natural to ask whether the conditions in Theorem 3.28 can be confirmed on a larger scale; that is, whether it can be proved that such a bound exists for an entire class of substitutions without computing the exponents explicitly. Fortunately, this is the case for a specific class, which we elaborate here. In particular, we will show the following result.

Theorem 4.1. Let $\varrho$ be a primitive, bijective constant-length substitution that is aperiodic and whose IDA $\mathcal{B}$ is Abelian. Then, all Lyapunov exponents of the outward iteration (25) of $\varrho$ are strictly positive, and the geometric realisation of the inflation tiling has singular diffraction. Moreover, all spectral measures of the dynamical spectrum are singular.

Throughout this section, we assume $\varrho$ to be an aperiodic, primitive, bijective substitution of length $L$ on an $n_{a}$-letter alphabet $\mathcal{A}_{n_{a}}$, with corresponding Fourier matrix $B(k)$ and associated IDA $\mathcal{B}$. Due to Fact 3.2, we make no distinction between $\mathcal{B}$ and the algebra generated by the digit matrices $\left\{D_{x} \mid x \in S_{T}\right\}$, which, in this setting, is the algebra generated by the matrix representation of the permutations $\left\{g_{0}, g_{1}, \ldots, g_{L-1}\right\}$, hence

$$
\mathcal{B}=\left\langle\left\{D_{x} \mid x \in S_{T}\right\}\right\rangle=\langle\Phi(G)\rangle,
$$


where $g_{r}$ is the inverse of the $r$-th column of the word vector $\left(\varrho\left(a_{i}\right)\right)_{1 \leqslant i \leqslant n_{a}}$, viewed as an element of the permutation group $\Sigma_{n_{a}}$ of $n_{a}$ elements, $G=\left\langle g_{0}, g_{1}, \ldots, g_{L-1}\right\rangle$ and $\Phi$ is the canonical representation via permutation matrices. This follows since $P^{T}=P^{-1}$ for any permutation matrix $P$, and hence $D_{x}=\left(\Phi\left(g_{x}\right)\right)^{T}=\Phi\left(g_{x}^{-1}\right)$. We call $G$ a generating subgroup for the algebra $\mathcal{B}$, where it is understood that $G$ is a subgroup of $\Sigma_{n_{a}}$. The primitivity condition on $\varrho$ translates to a condition on its generating subgroup as follows.

Lemma 4.2. Any generating subgroup $G$ for $\mathcal{B}$ must be a transitive subgroup of $\Sigma_{n_{a}}$.

Proof. Assume that $G$ is not transitive. Then, there are $a_{i}, a_{j} \in \mathcal{A}_{n_{a}}$ such that $\sigma\left(a_{i}\right)=a_{j}$ cannot hold for any $\sigma \in G$. Consequently, the representation matrices will be 0 in positions $i, j$, as are all linear combinations of them, and hence all elements of $\mathcal{B}$ by Fact 3.2.

Now, this implies that $a_{j}$ can never appear in any word of the form $\varrho^{n}\left(a_{i}\right)$ with $n \in \mathbb{N}$. This contradicts the assumed primitivity of $\varrho$, and our claim follows.

The following property of Abelian subgroups of $\Sigma_{n_{a}}$ is well known; see [54, Cor. 10.3.3 and Thm. 10.3.4].

Fact 4.3. Any transitive Abelian subgroup of $\Sigma_{n}$ must be of order $n$. So, if $G$ is an Abelian subgroup of $\Sigma_{n_{a}}$ that is generating for the IDA $\mathcal{B}$ of $\varrho$, it must be of order $n_{a}$.

Bijective substitutions have a rich structure due to the algebraic properties of their columns. These can be exploited to shed light on the multiplicity and mutual singularity of the spectral measures of the associated dynamical system; see [15, 51]. When the generating group is Abelian, these measures can be written down explicitly as Riesz products of polynomials arising from the characters $\rho \in \widehat{G}$ evaluated on the columns of $\varrho$. The following important result (actually, also its higher-dimensional analogue) was outlined in [51], and was formally proved in [15]. For binary block substitutions, it also follows from [30, 31], and it was shown in [4] by a different method.

Theorem 4.4 ([15, Thm. 4.19]). Any primitive, bijective constant-length substitution that is aperiodic and Abelian has purely singular dynamical spectrum.

In what follows, we prove that Theorem 3.28 holds for this class, thus giving an independent proof of Theorem 4.4 by yet another method, which also extends to higher-dimensional block substitutions [7]. Note that we impose no assumptions on the length or the height of $\varrho$.

Proof of Theorem 4.1. By assumption, the generating subgroup $G$ is Abelian, and all digit matrices $D_{x}$ commute with one another. Being permutation matrices, they are thus simultaneously diagonalisable, by a unitary matrix $U$ say. In this case, the diagonal entries of $U D_{x} U^{-1}$ are values of characters of $G$, written as $\rho_{i}(g)$. Note that the $\rho_{i}$ are the irreducible representations of $G$ because the latter is Abelian.

The matrix representation we start from is completely reducible by standard results [38], as a sum of one-dimensional irreducible representations, which are the group characters in this 
case. In its diagonalised version, all values that occur are thus roots of unity, so $\left|\rho_{i}(g)\right|=1$ for all $i$ and all $g \in G$. If $\varrho$ is of length $L$, the eigenvalues of $B(k)$ are then of the form

$$
\beta_{j}(k)=\sum_{m=0}^{L-1} \overline{\rho_{j}\left(g_{m}\right)} u^{m},
$$

which is a polynomial in $u=\mathrm{e}^{2 \pi \mathrm{i} k}$ of degree $L-1$ whose coefficients are all on the unit circle.

Since we are dealing with a constant-length substitution, $B^{(n)}(k)$ is 1-periodic and defines a matrix cocycle over the compact dynamical system given by $k \mapsto L k$ modulo 1 on $[0,1)$, which is ergodic with respect to Lebesgue measure. Oseledec's multiplicative ergodic theorem, compare [14,60], then guarantees the existence of the Lyapunov exponents of our matrix cocycle for a.e. $k \in \mathbb{R}$. Moreover, still for a set of full measure, the exponents are constant. They can be computed explicitly for each invariant subspace, where one obtains

$$
\chi_{j}=\log \sqrt{L}-\lim _{n \rightarrow \infty} \frac{1}{n} \sum_{\ell=0}^{n-1} \log \left|\beta_{j}\left(L^{\ell} k\right)\right| \underset{k \in \mathbb{R}}{\stackrel{\text { a.e. }}{=}} \log \sqrt{L}-\int_{0}^{1} \log \left|\beta_{j}(k)\right| \mathrm{d} k>0 .
$$

Here, the integral is strictly less than $\log \sqrt{L}$ because

$$
\exp \left(\int_{0}^{1} \log \left|\beta_{j}(k)\right| \mathrm{d} k\right)<\int_{0}^{1}\left|\beta_{j}(k)\right| \mathrm{d} k=\left\|\beta_{j}\right\|_{1}<\left\|\beta_{j}\right\|_{2}=\sqrt{L},
$$

where the first estimate follows from Jensen's inequality and is strict, as is the second because $\beta_{j}$ is not a monomial in $u$. The last equality is Parseval's identity; compare [46].

The diffraction measures of constant-length substitutions are closely related to the spectral measures of characteristic functions. More precisely, choosing $u_{i}=\delta_{i, \ell}$, the restriction of $\widehat{\gamma}$ to $[0,1)$ is the spectral measure of the characteristic function for the presence of a tile (letter) of type $\ell$ at 0 ; see [12] for details. Since our system is self-similar, this argument extends to characteristic functions of supertiles by a simple scaling argument.

Now, by [51, Prop. 7.2] or [15, Thm. 4.4], a spectral measure of maximal type can be constructed by a linear combination of spectral measures of characteristic functions of tiles and supertiles. But if none of them comprise an absolutely continuous component, the spectral measure of maximal type must be singular as well.

Remark 4.5. The integral to which the second summand of $\chi_{j}$ converges is known to be the logarithmic Mahler measure of the polynomial $\beta_{j}$, which we denote by $\mathfrak{m}\left(\beta_{j}\right)$. Here, the logarithmic Mahler measure of a (complex) polynomial $p$ is defined as

$$
\mathfrak{m}(p):=\int_{0}^{1} \log \left|p\left(\mathrm{e}^{2 \pi \mathrm{i} t}\right)\right| \mathrm{d} t .
$$

When $p(z)=a \prod_{i=1}^{n}\left(z-\alpha_{i}\right)$ is a non-zero polynomial of degree $n$, one has

$$
\mathfrak{m}(p)=\log |a|+\sum_{i=1}^{n} \log \left(\max \left\{1,\left|\alpha_{i}\right|\right\}\right)
$$

by Jensen's formula. In particular, for monic polynomials, $\mathfrak{m}(p)$ only depends on the roots of $p$ that lie outside the unit circle; see [27] for general background. 
This connection provides an effective tool to obtain good upper bounds of Lyapunov exponents for constant-length substitutions, in particular Abelian ones; compare [8]. This step conveniently generalises to higher-dimensional analogues of Abelian substitutions, ${ }^{6}$ then called Abelian block substitutions; compare [7]. With that, one completely recovers Bartlett's singularity result [15], for any dimension; see Theorem 5.10 below.

Example 4.6. Consider $\varrho^{2}$ with $\varrho$ from Example 3.8, which is a substitution on $\mathcal{A}_{4}$ with associated generating subgroup $G=C_{2} \times C_{2}$. The Fourier matrix $B(k)$ reads

$$
B(k)=\left(\begin{array}{cccc}
1 & u^{3} & u^{2} & u \\
u^{3} & 1 & u & u^{2} \\
u^{2} & u & 1 & u^{3} \\
u & u^{2} & u^{3} & 1
\end{array}\right),
$$

where $u=\mathrm{e}^{2 \pi \mathrm{i} k}$, while the corresponding eigenvalues are

$$
\begin{array}{ll}
\beta_{1}(k)=1-u-u^{2}+u^{3}, & \beta_{2}(k)=1+u-u^{2}-u^{3}, \\
\beta_{3}(k)=1-u+u^{2}-u^{3}, & \beta_{4}(k)=1+u+u^{2}+u^{3},
\end{array}
$$

with corresponding eigenvectors that are $k$-independent. These four polynomials are products of cyclotomic polynomials, and hence $\mathfrak{m}\left(\beta_{j}\right)=0$ for $1 \leqslant j \leqslant 4$. This results in a degenerate Lyapunov spectrum for a.e. $k \in \mathbb{R}$, and hence in $\chi^{B}=0<\log \sqrt{L}=\log (2)$.

Determining which substitutions have the same Lyapunov exponents is generally difficult, especially since the equality of Mahler measures, which only depend on the roots of a polynomial outside the unit circle, does not imply that they come from the same polynomial. However, as we shall see in the next result, a certain dichotomy gives rise to families of substitutions that share the same Lyapunov spectrum (before normalisation).

Theorem 4.7. Consider the $n_{a}$-letter constant-length substitution $\varrho: a_{i} \mapsto w_{i}$, with $\left|w_{i}\right|=L$ for all $i$, and assume that the columns are either bijective or constant. Suppose further that the group $G^{\prime}$ generated by the bijective columns is Abelian (but not necessarily transitive in $\left.\Sigma_{n_{a}}\right)$. Then, all Lyapunov exponents associated to @ are strictly positive.

Remark 4.8. We stress that the set of constant-length substitutions satisfying the conditions of Theorem 4.7 is a subset of the substitutions with at least one constant column (also known as a coincidence). Such substitutions, by Dekking's criterion [24], have pure point spectrum. What we have confirmed here, using our method via Lyapunov exponents, is the singularity of the spectrum for this specific subset. Though this is a weaker result, it is interesting in its own right, as it extends to other cases where Dekking's criterion yields no answer.

Proof of Theorem 4.7. From the premise, the Fourier matrix of $\varrho$ can be decomposed into

$$
B(k)=B_{\mathrm{b}}(k)+B_{\mathrm{c}}(k),
$$

\footnotetext{
${ }^{6}$ We refer to Example 5.12 in the next section for an illustration.
} 
where $B_{\mathrm{b}}(k)$ and $B_{\mathrm{c}}(k)$ are generated by the bijective and constant columns, respectively. This gives a partition of the positions as $\{0, \ldots, L-1\}=S_{\mathrm{b}} \cup S_{\mathrm{c}}$. The idea of the proof now is to show that all eigenvalues of $B_{\mathrm{b}}(k)$ except one (and their corresponding eigenvectors) are essentially inherited by $B(k)$. We begin by illustrating how this works for cases when $G^{\prime}$ is transitive, and later describe what changes in the case when it is not.

It follows from Theorem 4.1 that $B_{\mathrm{b}}(k)$ has $n_{a}$ linearly independent eigenvectors that do not depend on $k$. Furthermore, $n_{a}-1$ of them have a component sum equal to zero, with the remaining eigenvector being $v_{n_{a}}=(1,1, \ldots, 1)^{T}$. This property follows from the facts that these eigenvectors can directly be constructed from the character table of $G^{\prime}$ and that $\sum_{g \in G} \rho(g)=0$ for all irreducible representations $\rho \in \widehat{G^{\prime}}$ except the trivial one.

Consider any eigenvector $v$ of $B_{\mathrm{b}}(k)$, with eigenvalue $\beta(k)$ say, with zero component sum. Observe that we can write $B_{\mathrm{c}}(k)$ as

$$
B_{\mathrm{c}}(k)=\sum_{z \in S_{\mathrm{c}}} \mathrm{e}^{2 \pi \mathrm{i} z k} R_{a(z)}, \quad 1 \leqslant a(z) \leqslant n_{a},
$$

where the matrix $R_{m}$ has entries 1 in the $m$-th row and 0 everywhere else. Consequently, $R_{m} v=0$ for all $1 \leqslant m \leqslant n_{a}$, which implies $B_{\mathrm{c}}(k) v=0$. But this means that $v$ is also an eigenvector of $B(k)$, with the same eigenvalue $\beta(k)$.

As in Theorem 4.1, the eigenvalues of $B_{\mathrm{b}}$ can be written in terms of the characters of $G^{\prime}$,

$$
\beta_{j}(k)=\sum_{m \in S_{\mathrm{b}}} \overline{\rho_{j}\left(g_{m}\right)} u^{m},
$$

which is always a polynomial in $u=\mathrm{e}^{2 \pi \mathrm{i} k}$ of degree at most $L-1$. All its coefficients are either 0 or have modulus 1. Parseval's equation then once again guarantees that the Lyapunov exponents arising from these eigenvalues are strictly less than $\log \sqrt{L}$. The maximal Lyapunov exponent is achieved for some $j$, which in turn satisfies

$$
\chi^{B}=\chi_{j}=\mathfrak{m}\left(\beta_{j}\right)<\log \sqrt{L} .
$$

The $n_{a}-1$ exponents shared by $B$ and $B_{\mathrm{b}}$ clearly satisfy this bound. The idea is now to invoke Lyapunov forward regularity to show that the last exponent is zero, which is done prior to (additively) normalising with $\log \sqrt{L}$. This will confirm that $B$ and $B_{\mathrm{b}}$ indeed share the same set of exponents.

To this end, we note that the $n_{a}$-th eigenvalue of $B(k)$ is $\beta_{n_{a}}^{\prime}(u)=\sum_{m=0}^{L-1} u^{m}$, which easily follows from the trace formula. By Lyapunov forward regularity [14], we see that the sum of the exponents under the outward iteration (25), without the prefactor $\sqrt{\lambda}$, is given by

$$
\begin{aligned}
\sum_{m=1}^{n_{a}} \chi_{m}^{\prime} & =-\int_{0}^{1} \log |\operatorname{det}(B(k))| \mathrm{d} k=-\sum_{m=1}^{n_{a}} \int_{0}^{1} \log \left|\beta_{m}^{\prime}(k)\right| \mathrm{d} k \\
& =\chi_{1}^{\prime}+\chi_{2}^{\prime}+\cdots+\chi_{n_{a}-1}^{\prime}-\mathfrak{m}\left(\beta_{n_{a}}^{\prime}\right),
\end{aligned}
$$

from which it is clear that $\chi_{n_{a}}^{\prime}=-\mathfrak{m}\left(\beta_{n_{a}}^{\prime}\right)=0$ since $\beta_{n_{a}}^{\prime}$ is cyclotomic. This completes the argument for the transitive case. 
When $G^{\prime}$ fails to be transitive, we can still use the decomposition $B=B_{\mathrm{b}}+B_{\mathrm{c}}$, where $B_{\mathrm{b}}$ now has to be put into block diagonal form via some elementary matrix operations that partition $\mathcal{A}_{n_{a}}=\left\{a_{1}, \ldots, a_{n_{a}}\right\}$ into orbits of $G^{\prime}$. A particularly useful decomposition of $G^{\prime}$ is $G^{\prime} \simeq G_{1}^{\prime} \times \cdots \times G_{s}^{\prime}$, wherein each subgroup $G_{\ell}^{\prime}$ (which can be the trivial subgroup) acts transitively on the $s$ orbits in $\mathcal{A}_{n_{a}}$. Furthermore, each nontrivial $G_{\ell}^{\prime}$ can be written as a finite product of cyclic groups by the fundamental theorem of finite Abelian groups. This also means that the digit matrices afford the splitting

$$
D_{m}=\Phi\left(g_{m}^{-1}\right)=\bigoplus_{\ell=1}^{s} \overline{\Phi_{\ell}\left(g_{m}^{(\ell)}\right)}
$$

with $g_{m}=\bigoplus_{\ell=1}^{s} g_{m}^{(\ell)}$, where $\Phi_{\ell}$ is the permutation representation on $G_{\ell}^{\prime}$.

With this, we recover the eigenvalues of $B_{\mathrm{b}}$ from each block as

$$
\beta_{j}^{(\ell)}(k)=\sum_{m \in S_{\mathrm{b}}} \overline{\rho_{j}^{(\ell)}\left(g_{m}^{(\ell)}\right)} u^{m},
$$

where $\rho_{j}^{(\ell)}$ is an irreducible character of $G_{\ell}^{\prime}$. An immediate consequence is that $\sum_{m \in S_{\mathrm{b}}} u^{m}$ has multiplicity $s$ as an eigenvalue of $B_{\mathrm{b}}$ (corresponding to different eigenvectors) since all blocks naturally admit the trivial representation. Note that non-transitivity in conjunction with primitivity of the substitution implies that at least one coincidence must be present, which implies $\operatorname{card}\left(S_{\mathrm{b}}\right)<L$.

Similar to the transitive case, any eigenvector of $B_{\mathrm{b}}$ with zero component sum remains an eigenvector of $B$, with the same eigenvalue. All but one copy of the polynomial $\sum_{m \in S_{\mathrm{b}}} u^{m}$ also remain eigenvalues, but this time with the corresponding eigenvectors being linear combinations of eigenvectors from different blocks. Finally, the uninherited eigenvalue (the one with a $k$-dependent eigenvector) is the cyclotomic polynomial $\sum_{m=0}^{L-1} u^{m}$, which can be computed from the trace. It is easy to see that the same arguments unambiguously apply as in the transitive case, since the eigenvalues are polynomials in $u$ with coefficients of unit modulus.

At this point, some examples are in order.

Example 4.9 $\left(\mathcal{A}_{3}\right.$, transitive, $\left.G^{\prime} \simeq C_{3}\right)$. Consider the substitution $\varrho_{3}$, with Fourier matrix $B_{3}(k)$, given by

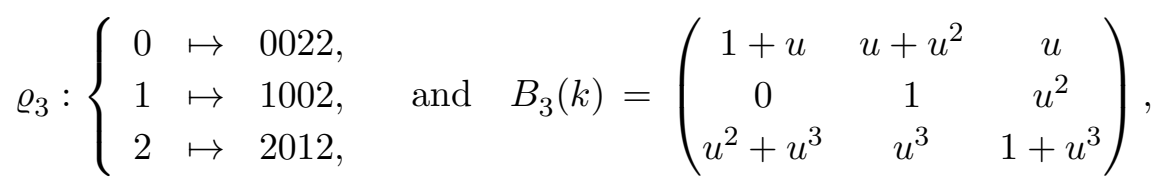

where $u=\mathrm{e}^{2 \pi \mathrm{i} k}$ as usual. The eigenvalues and eigenvectors of $B_{3}(k)$, which derive from the digit matrices that generate the Abelian IDA, are given by

$$
\begin{aligned}
& \beta_{1}(k)=1+\omega^{2} u^{2} \quad \text { with } \quad v_{1}=\left(\omega^{2}, \omega, 1\right)^{\mathrm{T}}, \\
& \beta_{2}(k)=1+\omega u^{2} \quad \text { with } \quad v_{2}=\left(\omega, \omega^{2}, 1\right)^{\mathrm{T}},
\end{aligned}
$$


where $\omega=\mathrm{e}^{\frac{2 \pi \mathrm{i}}{3}}$. The third eigenvalue is given by $\beta_{3}(k)=1+u+u^{2}+u^{3}$. The logarithmic Mahler measures $\mathfrak{m}\left(\beta_{i}\right)$ are all zero since the respective roots all lie on the unit circle. This implies that the (almost surely constant) exponents are $\chi_{j}=\log (2)-\mathfrak{m}\left(\beta_{j}\right)=\log (2)$ and thus all strictly positive.

Example $4.10\left(\mathcal{A}_{4}\right.$, non-transitive, $\left.G^{\prime} \simeq C_{2} \times C_{2}\right)$. The converse of Fact 4.3 is not true. There are Abelian subgroups of $\Sigma_{n}$ of order $n$ that are not transitive. In $\Sigma_{4}$, there are seven subgroups isomorphic to Klein's 4-group $C_{2} \times C_{2}$, only three of which are transitive. Here, we select a substitution where $G^{\prime}$ has two disjoint orbits. Consider the substitution $\varrho_{V}$, alongside with its corresponding Fourier matrix,

$$
\varrho_{V}:\{\begin{array}{rll}
0 & \mapsto & 0112, \\
1 & \mapsto & 1012, \\
2 & \mapsto & 3212, \\
3 & \mapsto 2312,
\end{array} \quad \text { and } \quad B(k)=\underbrace{\left(\begin{array}{cccc}
1 & u & 0 & 0 \\
u & 1 & 0 & 0 \\
0 & 0 & u & 1 \\
0 & 0 & 1 & u
\end{array}\right)}_{B_{\mathrm{b}}}+\underbrace{\left(\begin{array}{cccc}
0 & 0 & 0 & 0 \\
u^{2} & u^{2} & u^{2} & u^{2} \\
u^{3} & u^{3} & u^{3} & u^{3} \\
0 & 0 & 0 & 0
\end{array}\right)}_{B_{\mathrm{c}}} \text {. }
$$

The eigenvalues of $B$ that correspond to three $k$-independent eigenvectors of $B_{\mathrm{b}}$ are

$$
\begin{array}{ll}
\beta_{1}(k)=1-u, & v_{1}=(-1,1,0,0)^{T}, \\
\beta_{2}(k)=-1+u, & v_{2}=(0,0,-1,1)^{T}, \\
\beta_{3}(k)=1+u, & v_{3}=(-1,-1,1,1)^{T},
\end{array}
$$

with the last eigenvalue being $\beta_{4}(k)=1+u+u^{2}+u^{3}$. Here, one sees that $v_{3}$ is a linear combination of the eigenvectors from the two separate blocks of $B_{\mathrm{b}}$ corresponding to the same eigenvalue $\beta_{3}(k)$. The positivity of the Lyapunov exponents follows from the same arguments as in our previous examples.

As mentioned above in Theorem 3.36, it is a necessary criterion [17] for a primitive substitution $\varrho$ of constant length $L$ to have an absolutely continuous component in its dynamical spectrum that the substitution matrix of $\varrho$ must have an eigenvalue of modulus $\sqrt{L}$. One can easily check that the substitution matrix $M=B(0)$ of $\varrho_{V}$ has eigenvalues $\{4,2,0,0\}$, so it satisfies the criterion. However, $\varrho_{V}$ contains coincidences, and hence has pure point spectrum by Dekking's criterion [24]. The absence of AC spectral components is also rederived here via the positivity of the Lyapunov exponents.

Another example that satisfies the $\sqrt{L}$-criterion but evades AC spectral measures was analysed in [20]. Based on Bartlett's approach, it was shown to have singular continuous spectrum. The absence of AC spectral components was independently shown via Lyapunov exponents in [7]. Let us comment on how one can systematically construct examples that satisfy the $\sqrt{\lambda}$-criterion, but do not have absolutely continuous spectrum.

In general, one can begin with a (non-primitive) substitution of length $L$ in $n_{a}$ letters, whose columns are bijective and whose generating subgroup $G^{\prime}$ is a non-transitive subgroup of $\Sigma_{n_{a}}$. From the proof of Theorem $4.7, \beta(k)=1+u+\ldots+u^{L-1}$ is always an eigenvalue of $B_{\mathrm{b}}$, and at least one copy of it survives to be an eigenvalue of $B$, which means that $\beta(0)=L$ 
is an eigenvalue of $M=B(0)$ of the new substitution formed by adding coincidences. One can then choose to add appropriate columns, so that the resulting substitution is primitive, and enough columns, so that it is of length $L^{2}$.

\section{INFLATION TILINGS IN HIGHER DIMENSIONS}

At first sight, Theorem 3.28 and the methods employed to derive it appear as a onedimensional affair, which fortunately is not the case. In fact, it is precisely the separation of the geometry of the underlying space from the geometry of the inflation structure via the Fourier matrix that can be transferred to higher dimensions, which is one of the key advantages of the underlying geometric self-similarity.

The general setting for inflation tilings in $\mathbb{R}^{d}$ is as follows. We assume we are given $n_{a}$ prototiles $\mathcal{T}_{1}, \ldots, \mathcal{T}_{n_{a}}$ (that is, tiles up to translations), together with an expansive linear map $Q$ such that, for each $i$, the image $Q\left(\mathcal{T}_{i}\right)$ is a union of non-overlapping translates of prototiles. This is called a stone inflation in [5], and we will once again use $\varrho$ for the explicit inflation rule. The latter is called primitive if the corresponding incidence (or substitution) matrix is a primitive matrix. An important subclass is provided by inflations that lead to tilings of finite local complexity (FLC) with respect to translations. If one wants to go beyond this case, some extra precaution will be required.

Essentially as in the one-dimensional case, any primitive inflation defines a unique hull, called $\mathbb{Y}$, which can be obtained as the orbit closure of a fixed point tiling of $\varrho$ (or of $\varrho^{m}$ for a suitable $m \in \mathbb{N}$ ) under the translation action of $\mathbb{R}^{d}$. One has the following classic result; compare $[56,52,5,33,50]$ and references therein.

Fact 5.1. The hull $\mathbb{Y}$ of a primitive stone inflation $\varrho$ is compact in the local rubber topology, consists of a single LI class and gives rise to a minimal topological dynamical system $\left(\mathbb{Y}, \mathbb{R}^{d}\right)$. The latter is strictly ergodic, where the unique invariant probability measure is the patch frequency measure.

Remark 5.2. The term 'patch frequency' is the extension of a standard notion (word frequency) from symbolic dynamics to FLC tilings. Patch frequencies are used to define a translation-invariant measure on the tiling hull (equipped with the local topology) via cylinder sets; compare $[51,5]$.

Here, we also need the analogous concept for hulls of non-FLC tilings, now equipped with the local rubber topology $[10,5]$. The latter is a Fell topology and reduces to the local topology in the FLC case. The construction of a measure via cylinder sets now requires the consideration of patches (or clusters) up to $\varepsilon$-deformations for small $\varepsilon>0$. For primitive inflation rules with finitely many prototiles up to translations, the resulting properties are very similar to the FLC case; see $[33,44]$ for details.

Let us now assume that each prototile is equipped with a control point in such a way that any tiling in the hull $\mathbb{Y}$ is MLD with the corresponding set of control points. In this context, it is often convenient to distinguish (or colour) the points according to the prototile type. 
Unlike the situation in one dimension, there may not be an obvious or canonical choice for the control points. This does not matter much because the tiling hull and the corresponding set of point sets define topologically conjugate dynamical systems, wherefore we identify the two points of view. It will be clear from the context whether we speak about tilings or about their MLD control point sets.

Remark 5.3. Two (possibly coloured) point sets that are MLD need not (and generally do not) have the same pair correlations. Consequently, the diffraction measures will generally also differ, which reflects the fact that the diffraction measure neither is an invariant under topological conjugacy nor under metric isomorphism. Nevertheless, the type of the dynamical spectrum is invariant, and our approach via Lyapunov exponents thus aims at statements about the presence or absence of absolutely continuous spectral components.

Let us now develop the higher-dimensional analogue of the displacement matrix and how it can be used to derive Lyapunov exponents together with suitable estimates.

5.1. Displacement and Fourier matrices. Let $T_{i j}$ be the set of control point positions of tiles of type $i$ in the supertile of type $j$, relative to the control point position of the latter, where the location of the control points in the supertiles are determined by the action of the linear map $Q$. As before, $M=\operatorname{card}(T)$ is the incidence matrix, where $T=\left(T_{i j}\right)_{1 \leqslant i, j \leqslant n_{a}}$ is the set-valued displacement matrix. Likewise, $Q T=\left(Q T_{i j}\right)_{1 \leqslant i, j \leqslant n_{a}}$ with $Q T_{i j}:=\left\{Q(t) \mid t \in T_{i j}\right\}$ is the displacement matrix for the relative positions of supertiles in level-2 supertiles. Now, let $T^{(n)}$ denote the displacement matrix for the relative positions of tiles in the supertiles of level $n$, which is the displacement matrix for the inflation rule defined by $\varrho^{n}$. Clearly, $T^{(1)}=T$ and, adding the relative displacements by one additional inflation step, one recursively gets

$$
T_{i j}^{(n+1)}=\bigcup_{\ell=1}^{n_{a}}\left(T_{i \ell}+Q T_{\ell j}^{(n)}\right),
$$

where + denotes the Minkowski sum of two point sets, as defined by

$$
U+V=\{u+v \mid u \in U, v \in V\} .
$$

It is easy to check that $\operatorname{card}\left(T^{(n)}\right)=M^{n}$.

As before, with $\delta_{T}:=\left(\delta_{T_{i j}}\right)_{1 \leqslant i, j \leqslant n_{a}}$, the Fourier matrix of the inflation $\varrho$ is

$$
B(k):=\widehat{\widehat{\delta_{T}}}(k)=\widehat{\delta_{T}}(-k)
$$

with $k \in \mathbb{R}^{d}$. For each $k$, one has $B(k) \in \operatorname{Mat}\left(n_{a}, \mathbb{C}\right)$, and each matrix element of $B$, as a function of $k$, is a multivariate trigonometric polynomial.

Lemma 5.4. Let $\varrho$ define a primitive stone inflation, with finitely many translational prototiles and linear expansion $Q$. Further, let $B(k)$ be the Fourier matrix from Eq. (29). Then, for $n \in \mathbb{N}$, the Fourier matrix of $\varrho^{n}$ is given by

$$
B^{(n)}(k)=B(k) B\left(Q^{T} k\right) \cdots B\left(\left(Q^{T}\right)^{n-1} k\right)
$$

and satisfies $B^{(1)}=B$ together with $B^{(n+1)}(k)=B(k) B^{(n)}\left(Q^{T} k\right)$ for $n \in \mathbb{N}$. 


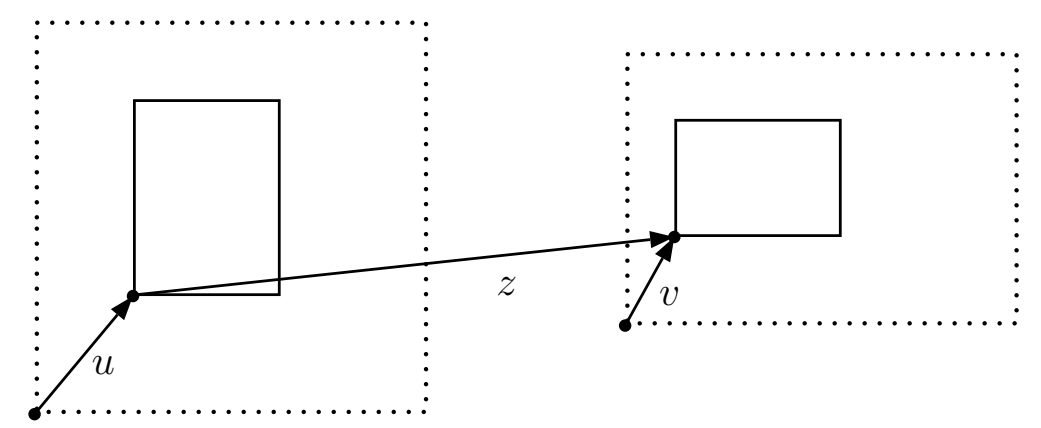

Figure 2. If the two tiles (solid lines) at distance $z$ have offsets $u$ and $v$ within their covering supertiles (dotted lines), the latter have distance $z+u-v$. Here, the distances are always defined via the control points of the tiles.

Proof. This is a consequence of Eq. (28) in conjunction with the observation that

$$
\mathrm{e}^{2 \pi \mathrm{i} k(u+Q v)}=\mathrm{e}^{2 \pi \mathrm{i} k u} \mathrm{e}^{2 \pi \mathrm{i} k Q v}=\mathrm{e}^{2 \pi \mathrm{i} k u} \mathrm{e}^{2 \pi \mathrm{i}\left(Q^{T} k\right) v} .
$$

Now, with $B^{(n)}(k)=\widehat{\delta_{T^{(n)}}}(-k)$, the structure of the Minkowski sum in (28) together with $\delta_{u+v}=\delta_{u} * \delta_{v}$ and the convolution theorem for Fourier transforms give the claim for $n=2$ by a simple calculation. The general formula with its recursive structure is then obvious.

5.2. Renormalisation relations for pair correlations. Let us begin with the case that the primitive inflation defines an FLC tiling hull. In complete analogy to one dimension, $\nu_{i j}(z)$ is defined as the relative frequency of finding a point of type $i$ and one of type $j$ separated by $z \in \mathbb{R}^{d}$, which is to be read as the vector from positions $i$ to $j$. In other words, one has

$$
\nu_{i j}(z)=\frac{\operatorname{dens}\left(\Lambda_{i} \cap\left(\Lambda_{j}-z\right)\right)}{\operatorname{dens}(\Lambda)},
$$

which is once again independent of the choice of $\Lambda$ from the hull, with $\operatorname{supp}\left(\nu_{i j}\right)=\Lambda_{j}-\Lambda_{i}$.

The exact renormalisation relations, first announced in [47], now read

$$
\nu_{i j}(z)=\frac{1}{|\operatorname{det}(Q)|} \sum_{m, n=1}^{n_{a}} \sum_{\substack{u \in T_{i m} \\ v \in T_{j n}}} \nu_{m n}\left(Q^{-1}(z+u-v)\right) .
$$

The derivation is once again based on recognisability in aperiodic inflation tilings, which holds here as well [57], and works exactly as in the one-dimensional case covered by Lemma 3.16; see Figure 2 for an illustration with block tiles whose lower left corners are chosen as their reference points, which explains the meaning of $u, v$ and $z$ in (31). The extension to also cover the periodic cases is a consequence of Remark 3.17.

With $\Upsilon_{i j}=\sum_{z} \nu_{i j}(z) \delta_{z}$, which can alternatively be defined as in Eq. (14), this leads to the corresponding relations for the pair correlation measures, namely

$$
\Upsilon_{i j}=\frac{1}{|\operatorname{det}(Q)|} \sum_{m, n=1}^{n_{a}} \widetilde{\delta_{T_{i m}}} * \delta_{T_{j n}} *\left(Q . \Upsilon_{m n}\right) .
$$


Its derivation from (31) is based on the same calculation as in the one-dimensional case. At this point, using the results from $[10,33]$ on Delone dynamical systems in the local rubber topology, it is not difficult to see that the pair correlation measures $\Upsilon_{i j}$ are well-defined, and that Eq. (32) is the correct relation among them, also in the non-FLC case, provided we start from a primitive inflation rule with finitely many translational prototiles. The crucial step here is that $T$ then still is a finite matrix of displacement sets.

Proposition 5.5. Let $\mathbb{Y}$ be the tiling hull of a primitive stone inflation with finitely many translational prototiles. Let $Q$ denote the corresponding expansive linear map and $T$ the displacement matrix. Then, the pair correlation measures $\Upsilon_{i j}$ are well defined and satisfy the renormalisation relations $(32)$.

Moreover, whenever $\mathbb{Y}$ is FLC, each $\Upsilon_{i j}$ is a pure point measure, and the pair correlation functions $\nu_{i j}$ defined in (30) satisfy the relations (31).

Remark 5.6. While the formulation in terms of a stone inflation is the closest analogue to the one-dimensional case, it is by no means necessary. Clearly, one can also consider a more general inflation scheme with a rule that guarantees the gapless and overlap-free cover of space in the limit. Various scenarios, and how they are related, are described in [5, Ch. 6], including many classic examples such as the Ammann-Beenker and the Penrose tilings, but also more complicated ones that can be reformulated as a stone inflation via fractiles (tiles with fractal boundaries); see [5, 32] for various examples. We leave further details to the interested reader.

5.3. Analysis after Fourier transform. Let $Q^{*}=\left(Q^{T}\right)^{-1}$ be the dual matrix and observe that $\widehat{Q \cdot \mu}=|\operatorname{det}(Q)|^{-1} Q^{*} . \widehat{\mu}$, as shown in [8, Lemma 2.5]. Then, Fourier transform turns relation (32) into

$$
\widehat{\Upsilon}_{i j}=\operatorname{det}(Q)^{-2} \sum_{m, n=1}^{n_{a}} B_{i m}(.) \overline{B_{j n}(.)}\left(Q^{*} \cdot \widehat{\Upsilon}_{m n}\right)
$$

By Lemma 3.22, which remains valid here without any change, Eq. (33) gives rise to three separate relations for the spectral types.

The analysis of the pure point part can be done in complete analogy to Section 3.4 and leads to

$$
\boldsymbol{I}(k)=\operatorname{det}(Q)^{-2} \boldsymbol{A}(k) \boldsymbol{I}\left(Q^{T} k\right)
$$

together with the eigenvector relation $\boldsymbol{A}(0) \boldsymbol{I}(0)=\operatorname{det}(Q)^{2} \boldsymbol{I}(0)$. Since $|\operatorname{det}(Q)|$ is the $\mathrm{PF}$ eigenvalue of $M:=B(0)$, which is the inflation or incidence matrix of our system, the entries $I_{i j}(0)$ of $\boldsymbol{I}(0)$ once again satisfy $(21)$, so $I_{i j}(0)=\operatorname{dens}(\Lambda)^{-2} \operatorname{dens}\left(\Lambda_{i}\right) \operatorname{dens}\left(\Lambda_{j}\right)$.

Likewise, when we again represent the absolutely continuous part of $\widehat{\Upsilon}$ by the vector $\boldsymbol{h}$ of Radon-Nikodym densities, Lemma 3.26 still holds, now with the relation

$$
\boldsymbol{h}(k)=\frac{1}{|\operatorname{det}(Q)|} \boldsymbol{A}(k) \boldsymbol{h}\left(Q^{T} k\right) .
$$


Clearly, also the argument with the dimensional reduction can be applied here. Consequently, we may consider the cocycle $B^{(n)}(k)$ from Lemma 5.4 in conjunction with the Lyapunov exponents

$$
\chi^{B}(k):=\limsup _{n \rightarrow \infty} \frac{1}{n} \log \left\|B^{(n)}(k)\right\| \quad \text { and } \quad \chi_{\min }(k)=\log \sqrt{|\operatorname{det}(Q)|}-\chi^{B}(k) .
$$

A rather straight-forward generalisation of our previous proof in one dimension results in the following higher-dimensional counterpart.

Theorem 5.7. Let $\varrho$ be a primitive inflation rule in $\mathbb{R}^{d}$, with finitely many translational prototiles and expansive linear map $Q$. Let $B(k)$ be the corresponding Fourier matrix, with $\operatorname{det}(B(k)) \neq 0$ for at least one $k \in \mathbb{R}^{d}$. If there is an $\varepsilon>0$ such that

$$
\chi^{B}(k) \leqslant \log \sqrt{|\operatorname{det}(Q)|}-\varepsilon
$$

holds for a.e. $k \in \mathbb{R}^{d}$, where $\chi^{B}(k)$ is the maximal Lyapunov exponent from (34), the diffraction measure of the system cannot have an absolutely continuous part.

In analogy to the one-dimensional case, $\chi_{\min }(k)>0$ for a subset of full measure within an open neighbourhood of 0 already suffices to rule out an absolutely continuous diffraction component. In fact, we can also repeat the proof of Theorem 3.34, with minor modifications, so that we obtain the following result.

Corollary 5.8. Let $\varrho$ be a primitive inflation rule with the conditions as in Theorem 5.7. Then, one has $\chi_{\min }(k) \geqslant 0$ for a.e. $k \in \mathbb{R}^{d}$. If the system displays a non-trivial diffraction component of absolutely continuous type, one must have $\chi_{\min }(k)=0$ for a subset of $\mathbb{R}^{d}$ of positive measure, which has full measure when $\chi_{\min }(k)$ is constant for a.e. $k \in \mathbb{R}^{d}$.

Let us now apply the theory to two planar examples of rather different nature. Further cases are discussed in detail in [7].

5.4. Block substitutions. Here, we consider unit cubes of finitely many types, say white and black in the binary case, together with a diagonal matrix $Q=\operatorname{diag}\left(q_{1}, \ldots, q_{d}\right)$, where all $q_{i} \geqslant 2$ for expansiveness. Using the key argument around Jensen's inequality and Parseval's equation from the proof of Theorem 4.1, it is not difficult to prove the following higherdimensional version of $[46$, Cor. 9]; see $[7,48]$ for details and further consequences.

Theorem 5.9. Let $\varrho$ be a primitive, binary block substitution in d dimensions, with expansion matrix $Q=\operatorname{diag}\left(q_{1}, \ldots, q_{d}\right)$ where $q_{i} \geqslant 2$ for $1 \leqslant i \leqslant d$. Then, the minimal Lyapunov exponent, for a.e. $k \in \mathbb{R}^{d}$, is bounded away from 0 , and we have absence of absolutely continuous diffraction. Moreover, all dynamical spectral measures are singular.

Likewise, we easily obtain the following version of Theorem 4.1 in higher dimensions.

Theorem 5.10. Let $\varrho$ be a primitive, bijective block substitution in d dimensions, whose IDA is Abelian, with $Q$ as in Theorem 5.9. Then, $\chi_{\min }(k)>0$ holds for a.e. $k \in \mathbb{R}^{d}$, and we have absence of absolutely continuous diffraction. As before, all spectral measures are singular. 
Remark 5.11. In both theorems, we are in the situation that our cocycle is effectively defined over a compact dynamical system, namely the one induced by the action of $Q$ on the $d$-torus. Consequently, by Oseledec's theorem, we know that the Lyapunov exponents almost surely exist as limits, and are constant on a set of full measure.

Also, in both cases, we know that the treatment of the diffraction measure gives access to a representative spectral measure of maximal type, via the lookup functions of tiles and supertiles at the origin, and thus determines its spectral type as well. In this sense, the answer for these cases is complete.

Let us illustrate Theorem 5.10 with a bijective ternary block substitution that also relates to our previous treatment of Abelian substitutions.

Example 5.12 (Planar block substitution with three tiles). Consider the inflation rule
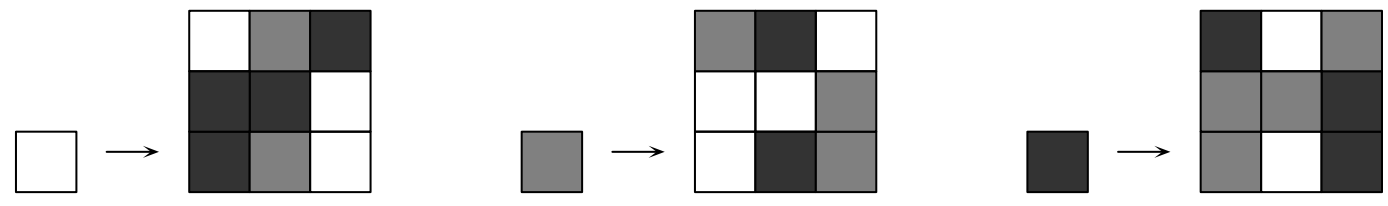

which is clearly primitive and bijective, with $Q=\operatorname{diag}(3,3)$. By inspection, one verifies that the permutation subgroup is isomorphic to $C_{3}$.

Its Fourier matrix reads

$$
B\left(k_{1}, k_{2}\right)=\left(\begin{array}{ccc}
x^{2}(1+y)+y^{2} & 1+y+x y+x^{2} y^{2} & x\left(1+y^{2}\right) \\
x\left(1+y^{2}\right) & x^{2}(1+y)+y^{2} & 1+y+x y+x^{2} y^{2} \\
1+y+x y+x^{2} y^{2} & x\left(1+y^{2}\right) & x^{2}(1+y)+y^{2}
\end{array}\right)
$$

with $x=\mathrm{e}^{2 \pi \mathrm{i} k_{1}}$ and $y=\mathrm{e}^{2 \pi \mathrm{i} k_{2}}$. The eigenvalues of the Fourier matrix are given by

$$
\begin{aligned}
& \beta_{1}\left(k_{1}, k_{2}\right)=\left(1+x+x^{2}\right)\left(1+y+y^{2}\right), \\
& \beta_{2}\left(k_{1}, k_{2}\right)=\left(x^{2}+x^{2} y+y^{2}\right)+\omega\left(1+y+x y+x^{2} y^{2}\right)+\omega^{2}\left(x+x y^{2}\right), \\
& \beta_{3}\left(k_{1}, k_{2}\right)=\left(x^{2}+x^{2} y+y^{2}\right)+\omega^{2}\left(1+y+x y+x^{2} y^{2}\right)+\omega\left(x+x y^{2}\right),
\end{aligned}
$$

where $\omega=\mathrm{e}^{\frac{2 \pi \mathrm{i}}{3}}$. As polynomials in two variables with complex coefficients, all of them are of height 1, and have logarithmic Mahler measures strictly less than $\log \sqrt{|\operatorname{det}(Q)|}=\log (3)$. In line with Theorem 5.7 and the comment following it, we thus see that the diffraction measures are singular, as are all spectral measures of the dynamical spectrum.

It is clear that there is an abundance of similar examples. As long as the permutation subgroup is Abelian, the corresponding bounds do not require $Q$ to be a homothety.

5.5. The Godrèche-Lançon-Billard tiling. Here, we briefly consider a non-PV inflation tiling of the plane that was described by Godrèche and Lançon in [36], following up an idea from Lançon and Billiard [40, Fig. 4]. It uses the two rhombuses of the Penrose tiling for a primitive inflation rule with expansive map $Q=\lambda R$, where

$$
\lambda=2 \cos \left(\frac{\pi}{10}\right)=\sqrt{\frac{1}{2}(5+\sqrt{5})} \approx 1.902
$$



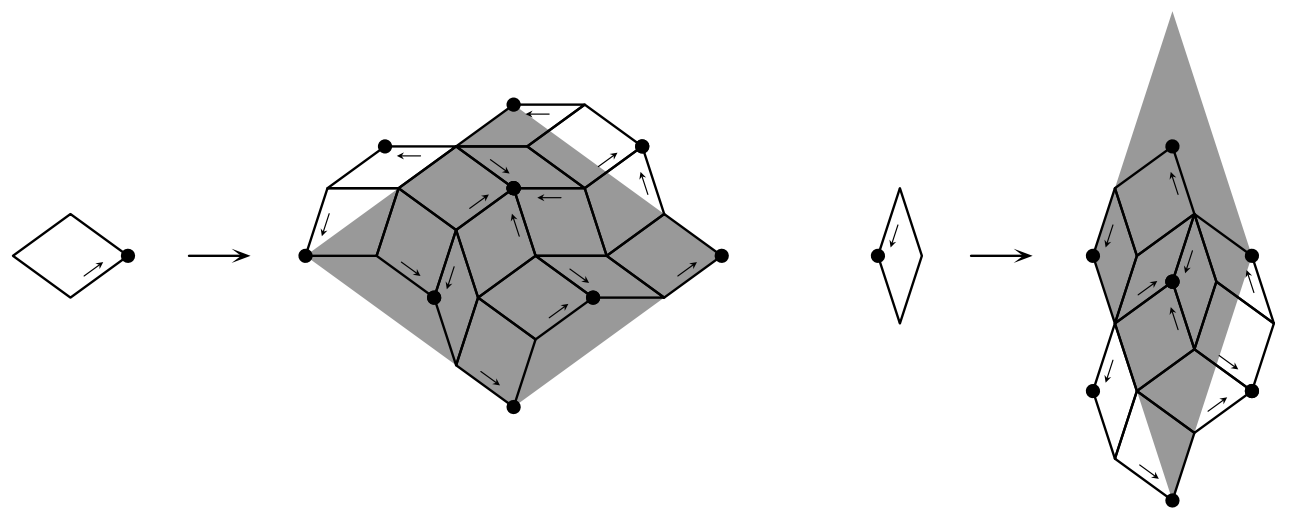

Figure 3. Modified inflation rule for the Godrèche-Lançon-Billiard tiling, with the control points marked by black dots.

and $R$ is a rotation through $\frac{\pi}{10}$; see [5, Sec. 6.5.1] for a detailed account, including its reformulation as a stone inflation with two fractiles, which we do not use here. This inflation rule defines an FLC tiling hull as usual, and any two elements are locally indistinguishable. We call the elements GLB tilings from now on. Note that $\lambda$ is not a PV number, so that one cannot have non-trivial eigenfunctions by [56], which also implies that the pure point part of the diffraction is trivial. As we shall see, we cannot have absolutely continuous diffraction either, which means that the diffraction is essentially singular continuous.

For our purposes here, it is a little easier to harvest the underlying fivefold rotation symmetry, and to work with the square of the inflation in conjunction with an added rotation through $\pi$, as illustrated in Figure 3. As one can quickly check, this modified rule defines the same tiling hull as the original one, ${ }^{7}$ now with the expansive map $\tilde{Q}=\lambda^{2} \mathbb{1}$. Each prototile contains an orientation vector as a marker, and the unique vertex it points to is chosen as its control point. Note that control points of different tiles can coincide, and that the vertex points of any GLB tiling differ in whether they carry a control point or not. Giving weight $\frac{1}{5}$ and $\frac{2}{5}$ to the control points of thick and thin rhombuses, respectively, one checks that these weights always add up to 1 in an occupied vertex point; compare [5, Figs. 6.27 and 6.28]. The relative frequency of control points among the vertices is $\frac{5-\sqrt{5}}{10} \approx 0.276393$.

Let $B^{(n)}(k)$ denote the Fourier matrix cocycle for the modified inflation rule. One can easily check numerically that $\operatorname{det}\left(B^{(1)}(k)\right) \neq 0$ for some $k=k_{0}$, hence by continuity for all $k$ in a small ball around $k_{0}$, and thus a.e. $k \in \mathbb{R}^{2}$ by analyticity. Then, to apply Theorem 5.7, we need to compare $\frac{1}{N} \mathbb{M}\left(\log \left\|B^{(N)}().\right\|\right)$ with $\frac{1}{2} \log (\operatorname{det}(\tilde{Q}))=2 \log (\lambda)$, with $\lambda$ as in (35). Employing the Frobenius norm, we may equivalently compare $\frac{1}{N} \mathbb{M}\left(\log \left\|B^{(N)}(.)\right\|_{\mathrm{F}}^{2}\right)$ with $4 \log (\lambda) \approx 2.571862$. Here, $\left\|B^{(N)}(k)\right\|_{\mathrm{F}}^{2}$ is a non-negative trigonometric polynomial in $k$ that is also quasiperiodic and, for any fixed $N$, bounded away from 0 . Its logarithm is then a Bohr almost periodic function that can be represented as a section of a periodic function

\footnotetext{
${ }^{7}$ Some statistical data, such as the relative frequency of the prototiles or the frequency of vertices that carry a control point, can be extracted with standard Perron-Frobenius arguments from either rule.
} 
TABLE 1. Some upper bounds for $\chi^{B}(k)$ via means as explained in the main text, which are to be compared with the threshold value $4 \log (\lambda) \approx 2.571862$. The numerical error is less than 0.005 in all cases listed.

\begin{tabular}{|c|cccccccc|}
\hline$N$ & 6 & 7 & 8 & 9 & 10 & 11 & 12 & 13 \\
\hline$\frac{1}{N} \mathbb{M}\left(\log \left\|B^{(N)}(.)\right\|_{\mathrm{F}}^{2}\right)$ & 2.643 & 2.572 & 2.517 & 2.474 & 2.440 & 2.411 & 2.387 & 2.367 \\
\hline
\end{tabular}

on the 4-torus, and its mean is then given as an integral over $\mathbb{T}^{4}$. The numerical calculation leads to the values shown in Table 1 , and thus to the following conclusion.

Theorem 5.13. The Fourier transform $\widehat{\Upsilon}$ of the pair correlation measures for any GLB tiling with the control points as defined above is a vector of singular measures, and $\widehat{\Upsilon}$ is the same for all elements of the tiling hull.

For any $G L B$ tiling, with vertex set $\Lambda$, the measure $\omega=\sum_{x \in \Lambda} w(x) \delta_{x}$ with the coincidence weights $w(x)$ from above has singular diffraction with trivial pure point part.

Indeed, as mentioned above, we know that the pure point part is the trivial one. The singular diffraction measure $\widehat{\gamma_{\omega}}$ is thus of the form

$$
\widehat{\gamma_{\omega}}=I_{0} \delta_{0}+\left(\widehat{\gamma_{\omega}}\right)_{\mathrm{sc}} \quad \text { with } \quad I_{0}=\left(\frac{5-\sqrt{5}}{10} \operatorname{dens}(\Lambda)\right)^{2} \text {, }
$$

where $\operatorname{dens}(\Lambda)=\frac{1+\sqrt{5}}{5} \lambda=\frac{2}{5} \sqrt{5+2 \sqrt{5}} \approx 1.231$, if we work with rhombuses of unit edge length; compare [5, Cor. 9.1]. Now, it is not difficult to see that the vertex set with uniform weights is locally derivable from the pattern with the weights used above and vice versa, which means they are MLD. Although this will lead to different pair correlation and diffraction measures, their spectral type remains unchanged, with the following consequence.

Corollary 5.14. The diffraction measure of the uniform Dirac comb $\delta_{\Lambda}$ on the vertex set $\Lambda$ of any GLB tiling is singular, and of the form $\widehat{\gamma}=\operatorname{dens}(\Lambda)^{2} \delta_{0}+(\widehat{\gamma})_{\mathrm{sc}}$.

It remains to extend this result to the spectral measures of the corresponding translational dynamical system, where we expect the spectral measure of maximal type to be singular as well, with a trivial pure point part due to the constant eigenfunction.

\section{Appendix: The (skew) Kronecker product Algebra}

The structure of the correlation measures relies on some properties of the Kronecker product matrices

$$
\boldsymbol{A}(k):=B(k) \otimes \overline{B(k)},
$$

defined for $k \in \mathbb{R}$. Obviously, one has $\overline{\boldsymbol{A}(k)}=\boldsymbol{A}(-k)$ and $\operatorname{det}(\boldsymbol{A}(k))=|\operatorname{det}(B(k))|^{2 n_{a}}$.

In view of the structure of Eq. (36), let us now consider the $\mathbb{R}$-algebra $\mathcal{A}$ that is generated by the matrix family $\{\boldsymbol{A}(k) \mid k \in \mathbb{R}\}$. Due to the Kronecker product structure, $\mathcal{A}$ fails to be irreducible, no matter what the structure of the IDA $\mathcal{B}$ is. Let us explore this in some 
more detail. Let $V=\mathbb{C}^{n_{a}}$ and consider $W:=V \otimes_{\mathbb{C}} V$, the (complex) tensor product, which is a vector space over $\mathbb{C}$ of dimension $n_{a}^{2}$, but also one over $\mathbb{R}$, then of dimension $2 n_{a}^{2}$. In the latter setting, consider the involution $C: W \longrightarrow W$ defined by

$$
x \otimes y \longmapsto C(x \otimes y):=\overline{y \otimes x}=\bar{y} \otimes \bar{x}
$$

together with its unique extension to an $\mathbb{R}$-linear mapping on $W$. Observe that there is no $\mathbb{C}$-linear extension, because $C(a(x \otimes y))=\bar{a} C(x \otimes y)$ for $a \in \mathbb{C}$. With this definition of $C$, one finds for an arbitrary $k \in \mathbb{R}$ that

$$
\begin{aligned}
\boldsymbol{A}(k) C(x \otimes y) & =(B(k) \otimes \overline{B(k)})(\bar{y} \otimes \bar{x})=(B(k) \bar{y}) \otimes(\overline{B(k) x}) \\
& =C((B(k) \otimes \overline{B(k)})(x \otimes y))=C(\boldsymbol{A}(k)(x \otimes y)),
\end{aligned}
$$

so $C$ commutes with the linear map defined by $\boldsymbol{A}(k)$, for any $k \in \mathbb{R}$. The $\mathbb{R}$-linear mapping $C$ has eigenvalues \pm 1 and is diagonalisable, as follows from the unique splitting of an arbitrary $w \in W$ as $w=\frac{1}{2}(w+C(w))+\frac{1}{2}(w-C(w))$. So, our vector space splits as $W=W_{+} \oplus W_{-}$ into real vector spaces that are eigenspaces of $C$. Their dimensions are

$$
\operatorname{dim}_{\mathbb{R}}\left(W_{+}\right)=\operatorname{dim}_{\mathbb{R}}\left(W_{-}\right)=n_{a}^{2}
$$

since $W_{-}=\mathrm{i} W_{+}$with $W_{+} \cap W_{-}=\{0\}$. It is thus clear that $W_{+}$and $W_{-}$are invariant (real) subspaces for the $\mathbb{R}$-algebra $\mathcal{A}$.

Observe next that we have

$$
\boldsymbol{A}(k)=B(k) \otimes \overline{B(k)}=\sum_{x, y \in S_{T}} \mathrm{e}^{2 \pi \mathrm{i} k(x-y)} D_{x} \otimes D_{y}=\sum_{z \in \triangle_{T}} \mathrm{e}^{2 \pi \mathrm{i} k z} F_{z}
$$

where $\triangle_{T}:=S_{T}-S_{T}$ is the Minkowski difference, with $-\triangle_{T}=\triangle_{T}$, and

$$
F_{z}=\sum_{\substack{x, y \in S_{T} \\ x-y=z}} D_{x} \otimes D_{y} .
$$

In analogy to before, $F_{z}=F_{z^{\prime}}$ is possible for $z \neq z^{\prime}$. For instance, if $z=x-y$ with $x \neq y$ and $D_{x}=D_{y}$, one can get $F_{z}=F_{-z}$ if there is no other way to write $z$ as a difference of two numbers in $S_{T}$.

For $a \in \mathbb{C}$, one easily checks that $C \circ\left(a F_{z}\right)=\bar{a} C \circ F_{z}=\bar{a} F_{-z} \circ C$, which implies $[C, \boldsymbol{A}(k)]=0$ for all $k \in \mathbb{R}$, in line with our previous derivation. It is immediate that $\mathcal{A}$ is contained in the $\mathbb{R}$-algebra $\mathcal{A}_{F}$ that is generated by the matrices $\left\{F_{z}+F_{-z} \mid 0 \leqslant z \in \triangle_{T}\right\}$ together with $\left\{\mathrm{i}\left(F_{z}-F_{-z}\right) \mid 0 \leqslant z \in \triangle_{T}\right\}$, and an argument similar to the one previously used for $\mathcal{B}$ shows that $\mathcal{A}_{F} \subseteq \mathcal{A}$, hence $\mathcal{A}=\mathcal{A}_{F}$. Since $\operatorname{dim}_{\mathbb{C}}(\mathcal{B}) \leqslant n_{a}^{2}$, and since we generate the real algebra only after taking the Kronecker product, one has

$$
\operatorname{dim}_{\mathbb{R}}(\mathcal{A}) \leqslant n_{a}^{4}
$$

which is also clear from $\operatorname{dim}_{\mathbb{R}}\left(W_{+}\right)=n_{a}^{2}$. Moreover, one has the following result.

Lemma 5.15. Let $\varrho$ be a primitive inflation rule on an alphabet with $n_{a}$ letters, and assume that the IDA $\mathcal{B}$ of $\varrho$ is irreducible over $\mathbb{C}$. Then, the induced $\mathbb{R}$-algebra $\mathcal{A}$ is isomorphic with $\operatorname{Mat}\left(n_{a}^{2}, \mathbb{R}\right)$, and its action on the subspace $W_{+}$is irreducible as well, this time over $\mathbb{R}$. 
Proof. Here, $\mathcal{B}$ irreducible over $\mathbb{C}$ means $\mathcal{B}=\operatorname{Mat}\left(n_{a}, \mathbb{C}\right)$. With $\Gamma:=\mathcal{B} \otimes_{\mathbb{C}} \mathcal{B}$, where $\otimes_{\mathbb{C}}$ denotes the tensor product over $\mathbb{C}$, one has $\Gamma \simeq \operatorname{Mat}\left(n_{a}^{2}, \mathbb{C}\right)$ by standard arguments. Clearly, $\Gamma$ is a $\mathbb{C}$-algebra of dimension $n_{a}^{4}$, but also an $\mathbb{R}$-algebra, then of dimension $2 n_{a}^{4}$. Now, using the Kronecker product as representation of the tensor product, $M \otimes N \mapsto \bar{N} \otimes \bar{M}$ defines a mapping that has a unique extension to an automorphism $\sigma$ of $\Gamma$ as an $\mathbb{R}$-algebra.

Our $\mathbb{R}$-algebra $\mathcal{A}$ consists of all fixed points of $\sigma$, so $\mathcal{A}=\{Q \in \Gamma \mid \sigma(Q)=Q\}$. Employing the elementary matrices $E_{i j}$ from $\operatorname{Mat}\left(n_{a}, \mathbb{R}\right)$ together with $E_{i j, k \ell}:=E_{i k} \otimes E_{j \ell}$, we can give a basis of $\mathcal{A}$, seen as a vector space over $\mathbb{R}$, by

$$
\left\{\frac{1}{2}\left(E_{i j, k \ell}+E_{k \ell, i j}\right) \mid(i, j) \leqslant(k, \ell)\right\} \cup\left\{\frac{\mathrm{i}}{2}\left(E_{i j, k \ell}-E_{k \ell, i j}\right) \mid(i, j)<(k, \ell)\right\},
$$

where lexicographic ordering is used for the double indices. Note that the cardinalities are $\frac{1}{2} n_{a}^{2}\left(n_{a}^{2}+1\right)$ and $\frac{1}{2} n_{a}^{2}\left(n_{a}^{2}-1\right)$, which add up to $\operatorname{dim}_{\mathbb{R}}(\mathcal{A})=n_{a}^{4}$.

Next, observe that we can get $E_{i j, k \ell}$ and $E_{k \ell, i j}$ by a simple (complex) linear combination of $\frac{1}{2}\left(E_{i j, k \ell}+E_{k \ell, i j}\right)$ and $\frac{\mathrm{i}}{2}\left(E_{i j, k \ell}-E_{k \ell, i j}\right)$, and vise versa. Put together, this defines a (complex) inner automorphism of $\Gamma$. Observing that $\operatorname{Mat}\left(n_{a}^{2}, \mathbb{R}\right)=\{Q \in \Gamma \mid \bar{Q}=Q\}$, this construction can now be used to show that $\mathcal{A} \simeq \operatorname{Mat}\left(n_{a}^{2}, \mathbb{R}\right)$, which is a central simple algebra. Since $\mathcal{A} W_{+} \subseteq W_{+}$and $\operatorname{dim}_{\mathbb{R}}\left(W_{+}\right)=n_{a}^{2}$, the claimed irreducibility over the reals follows.

Note that all $F_{z}$ are non-negative, integer matrices. They clearly satisfy the relation $\sum_{z \in \triangle_{T}} F_{z}=\boldsymbol{A}(0)=M_{\varrho} \otimes M_{\varrho}$. Moreover, under some mild conditions, the spectral radius of $F_{0}$ is $\lambda$, while the other matrices $F_{z}$ have smaller spectral radius.

Let us come back to Eq. (36), which implies

$$
\|\boldsymbol{A}(k)\|_{\mathrm{F}}=\|B(k)\|_{\mathrm{F}}^{2} .
$$

If we consider the matrix cocycle defined by $\boldsymbol{A}^{(n)}(k)=B^{(n)}(k) \otimes \overline{B^{(n)}(k)}$, it is immediate that the maximal Lyapunov exponents, for all $k \in \mathbb{R}$, are related by

$$
\chi^{\boldsymbol{A}}(k)=2 \chi^{B}(k),
$$

which also holds for the higher-dimensional case with $k \in \mathbb{R}^{d}$. Clearly, one can now reformulate Theorems 3.28 and 5.7 in terms of $\chi^{A}$. In particular, one has the following reformulation of Theorem 3.34 and Corollary 3.35 and their higher-dimensional analogues.

Corollary 5.16. Let $\varrho$ be a primitive inflation rule in $\mathbb{R}^{d}$ with finitely many translational prototiles and expansive map $Q$. Let $B^{(n)}($.$) be its Fourier matrix cocycle, with \operatorname{det}(B(k)) \neq 0$ for at least one $k \in \mathbb{R}^{d}$, and $\boldsymbol{A}^{(n)}()=.B^{(n)}(.) \otimes \overline{B^{(n)}(.)}$ the corresponding Kronecker product cocycle. If the diffraction measure of the hull defined by @ contains a non-trivial absolutely continuous component, one has $\chi^{\boldsymbol{A}}(k)=\log |\operatorname{det}(Q)|$ for a subset of $\mathbb{R}^{d}$ of positive measure, which has full measure when $\chi^{\boldsymbol{A}}(k)$ is constant for a.e. $k \in \mathbb{R}^{d}$.

\section{ACKNOWLEDGEMENTS}

It is a pleasure to thank Frederic Alberti, Alan Bartlett, Scott Balchin, Natalie Frank, Uwe Grimm, Andrew Hubery, Robbie Robinson, Boris Solomyak and Nicolae Strungaru for 
helpful discussions. We also thank two anonymous reviewers for their thoughtful comments. This work was supported by the German Research Foundation (DFG), within the CRC 1283.

\section{REFERENCES}

[1] S. Akiyama, M. Barge, V. Berthé, J.-Y. Lee and A. Siegel, On the Pisot substitution conjecture, in [39], pp. 33-72.

[2] M. Baake, N.P. Frank, U. Grimm and E.A. Robinson, Geometric properties of a binary non-Pisot inflation and absence of absolutely continuous diffraction, Studia Math. 247 (2019) 109-154; arXiv: 1706.03976.

[3] M. Baake and F. Gähler, Pair correlations of aperiodic inflation rules via renormalisation: Some interesting examples, Topol. \& Appl. 205 (2016) 4-27; arXiv:1511.00885.

[4] M. Baake and U. Grimm, Squirals and beyond: Substitution tilings with singular continuous spectrum, Ergodic Th. \&3 Dynam. Syst. 34 (2014) 1077-1102; arXiv:1205.1384.

[5] M. Baake and U. Grimm, Aperiodic Order. Vol. 1: A Mathematical Invitation, Cambridge Univ. Press, Cambridge (2013).

[6] M. Baake and U. Grimm (eds.), Aperiodic Order. Vol. 2: Crystallography and Almost Periodicity, Cambridge University Press, Cambridge (2017).

[7] M. Baake and U. Grimm, Renormalisation of pair correlations and their Fourier transforms for primitive block substitutions, in Tiling and Discrete Geometry, eds. S. Akiyama and P. Arnoux, Springer, Berlin, in press; arXiv:1906.10484.

[8] M. Baake, U. Grimm and N. Mañibo, Spectral analysis of a family of binary inflation rules, Lett. Math. Phys. 108 (2018) 1783-1805; arXiv:1709.09083.

[9] M. Baake, A. Haynes and D. Lenz, Averaging almost periodic functions along exponential sequences, in [6], pp. 343-362; arXiv:1704.08120.

[10] M. Baake and D. Lenz, Dynamical systems on translation bounded measures: Pure point dynamical and diffraction spectra, Ergodic Th. \& Dynam. Syst. 24 (2004) 1867-1893;

arXiv:math.DS/0302231.

[11] M. Baake and D. Lenz, Spectral notions of aperiodic order, Discr. Cont. Dynam. Syst. S 10 (2017) 161-190; arXiv:1601.06629.

[12] M. Baake, D. Lenz and A.C.D. van Enter, Dynamical versus diffraction spectrum for structures with finite local complexity, Ergodic Th. \& Dynam. Syst. 35 (2015) 2017-2043; arXiv: 1307.7518.

[13] M. Baake and R.V. Moody, Weighted Dirac combs with pure point diffraction, J. Reine Angew. Math. (Crelle) 573 (2004) 61-94; arXiv:math.MG/0203030.

[14] L. Barreira and Y. Pesin, Nonuniform Hyperbolicity, Cambridge University Press, Cambridge (2007).

[15] A. Bartlett, Spectral theory of $\mathbb{Z}^{d}$ substitutions, Ergodic Th. E Dynam. Syst. 38 (2018) 12891341; arXiv: 1410.8106.

[16] C. Berg and G. Forst, Potential Theory on Locally Compact Abelian Groups, Springer, Berlin (1975).

[17] A. Berlinkov and B. Solomyak, Singular substitutions of constant length, Ergodic Th. ES Dynam. Syst., in press; arXiv: 1705.00899.

[18] A.I. Bufetov and B. Solomyak, On the modulus of continuity for spectral measures in substitution dynamics, Adv. Math. 260 (2014) 84-129; arXiv:1305.7373. 
[19] A.I. Bufetov and B. Solomyak, A spectral cocycle for substitution systems and translation flows, preprint arXiv: 1802.04783.

[20] L. Chan and U. Grimm, Spectrum of a Rudin-Shapiro-like sequence, Adv. Appl. Math. 87 (2017) 16-23; arXiv: 1611.04446.

[21] L. Chan, U. Grimm and I. Short, Substitution-based structures with absolutely continuous spectrum, Indag. Math. 29 (2018) 1072-1086; arXiv:1706.05289.

[22] A. Clark and L. Sadun, When size matters: Subshifts and their related tiling spaces, Ergodic Th. E Dynam. Syst. 23 (2003) 1043-57; arXiv:math.DS/0201152.

[23] D. Damanik, R. Sims and G. Stolz, Localization for one-dimensional, continuum, BernoulliAnderson models, Duke Math. J. 114 (2002) 59-100; arXiv:math-ph/0010016.

[24] F.M. Dekking, The spectrum of dynamical systems arising from substitutions of constant length, Z. Wahrscheinlichkeitsth. Verw. Geb. 41 (1978) 221-239.

[25] F. Durand, A characterization of substitutive sequences using return words, Discr. Math. 179 (1998) 89-101; arXiv:0807.3322.

[26] M. Einsiedler and T. Ward, Ergodic Theory - with a View towards Number Theory, GTM 259, Springer, London (2011).

[27] G. Everest and T. Ward, Heights of Polynomials and Entropy in Algebraic Dynamics, Springer, London (1999).

[28] A.-H. Fan, B. Saussol and J. Schmeling, Products of non-stationary random matrices and multiperiodic equations of several scaling factors, Pacific J. Math. 214 (2004) 31-54; arXiv:math.DS/0210347.

[29] N.P. Frank, Substitution sequences in $\mathbb{Z}^{d}$ with a nonsimple Lebesgue component in the spectrum, Ergodic Th. 83 Dynam. Syst. 23 (2003) 519-532.

[30] N.P. Frank, Multi-dimensional constant-length substitution sequences, Topol. \& Appl. 152 (2005) $44-69$.

[31] N.P. Frank, Introduction to hierarchical tiling dynamical systems, in Tiling and Discrete Geometry, eds. S. Akiyama and P. Arnoux, Springer, Berlin, in press; preprint arXiv: 1802.09956.

[32] D. Frettlöh, More inflation tilings, in [6], pp. 1-37.

[33] D. Frettlöh and C. Richard, Dynamical properties of almost repetitive Delone sets, Discr. Cont. Dynam. Syst. A 34 (2014) 531-556; arXiv:1210.2955.

[34] F. Gantmacher, Matrizentheorie, Springer, Berlin (1986).

[35] J. Gil de Lamadrid and L.N. Argabright, Almost periodic measures, Memoirs Amer. Math. Soc. 85 (1990) no. 428 (AMS, Providence, RI).

[36] C. Godrèche and F. Lançon, A simple example of a non-Pisot tiling with five-fold symmetry, $J$. Phys. I (France) 2 (1992) 207-220.

[37] A. Hof, On diffraction by aperiodic structures, Commun. Math. Phys. 169 (1995) 25-43.

[38] G. James and M. Liebeck, Representations and Characters of Groups, 2nd ed., Cambridge University Press, Cambridge (2001).

[39] J. Kellendonk, D. Lenz and J. Savinien (eds.), Mathematics of Aperiodic Order, Birkhäuser, Basel (2015).

[40] F. Lançon and L. Billiard, Two-dimensional system with a quasicrystalline ground state, J. Phys. (France) 49 (1988) 249-256.

[41] S. Lang, Algebra, rev. 3rd ed., Springer, New York (2002). 
[42] J.-Y. Lee, R.V. Moody and B. Solomyak, Pure point dynamical and diffraction spectra, Ann. Henri Poincaré 3 (2002) 1003-1018; arXiv:0910.4809.

[43] D. Lenz, Continuity of eigenfunctions of uniquely ergodic dynamical systems and intensity of Bragg peaks, Commun. Math. Phys. 287 (2009) 225-258; arXiv:math-ph/0608026.

[44] D. Lenz and N. Strungaru, Pure point spectrum for measure dynamical systems on locally compact Abelian groups, J. Math. Pures Appl. 92 (2009) 323-341; arXiv:0704. 2498.

[45] V. Lomonosov and P. Rosenthal, The simplest proof of Burnside's theorem on matrix algebras, Lin. Alg. Appl. 383 (2004) 45-47.

[46] N. Mañibo, Lyapunov exponents for binary substitutions of constant length, J. Math. Phys. 58 (2017) 113504:1-9; arXiv:1706.00451.

[47] N. Mañibo, Spectral analysis of primitive inflation rules, Oberwolfach Rep. 14 (2017) 2830-2832.

[48] N. Mañibo, Lyapunov Exponents in the Spectral Theory of Primitive Inflation Systems, PhD thesis, Bielefeld University (2019); available at https://pub.uni-bielefeld.de/record/2935972.

[49] R.V. Moody and N. Strungaru, Almost periodic measures and their Fourier transforms, in [6], pp. $173-270$.

[50] P. Müller and C. Richard, Ergodic properties of randomly coloured point sets, Can. J. Math. 65 (2013) 349-402; arXiv: 1005.4884.

[51] M. Queffélec, Substitution Dynamical Systems - Spectral Analysis, 2nd ed., LNM 1294, Springer, Berlin (2010).

[52] E.A. Robinson Jr., Symbolic dynamics and tilings of $\mathbb{R}^{d}$, Proc. Sympos. Appl. Math. 60 (2004) 81-119.

[53] W. Rudin, Fourier Analysis on Groups, Wiley, New York (1962).

[54] W.R. Scott, Group Theory, Prentice Hall, Englewood Cliffs, NJ (1964).

[55] B. Sing, Pisot Substitutions and Beyond, PhD thesis, Bielefeld University (2006); available at https://pub.uni-bielefeld.de/record/2302336.

[56] B. Solomyak, Dynamics of self-similar tilings, Ergodic Th. E D Dynam. Syst. 17 (1997) 695-738 and Ergodic Th. \& Dynam. Syst. 19 (1999) 1685 (erratum).

[57] B. Solomyak, Nonperiodicity implies unique composition for self-similar translationally finite tilings, Discr. Comput. Geom. 20 (1998) 265-278.

[58] N. Strungaru, On the Fourier analysis of measures with Meyer set support, preprint arXiv: 1807.03815.

[59] H.-R. Trebin (ed.), Quasicrystals - Structure and Physical Properties, Wiley-VCH, Weinheim (2003).

[60] M. Viana, Lectures on Lyapunov Exponents, Cambridge University Press, Cambridge (2013).

[61] A. Vince, Digit tiling of Euclidean space, in: M. Baake and R.V. Moody (eds.), Directions in Mathematical Quasicrystals, CRM Monograph Series, vol. 13, AMS, Providence, RI (2000), pp. 329-370.

FakUltät für Mathematik, Universität Bielefeld,

Postfach 100131, 33501 Bielefeld, Germany

E-mail address: \{mbaake, gaehler, cmanibo\}@math.uni-bielefeld.de 